\title{
What housing features should inform the development of housing solutions for adults with neurological disability?: A systematic review of the literature
}

\begin{abstract}
Despite the recent emphasis in Australian political, academic, and legislative narratives to more actively promote real housing choice for people with high healthcare and support needs, there is a lack of understanding regarding the specific housing features that might constitute better housing solutions for this population. Inclusive housing provision in Australia rightly emphasises safety and accessibility issues but often fails to incorporate factors related to broader psychosocial elements of housing such as dwelling location, neighbourhood quality, and overall design. While the importance of these broader elements appears obvious, it is not yet clear what specific housing features relate to these elements and how they might contribute to housing solutions for people with high healthcare and support needs. For individuals with complex neurological conditions such as brain injury or cerebral palsy, who require maximum support on a daily basis yet want to live independently and away from a primary care hospital or health facility, a more detailed understanding of the housing features that might influence design and development is needed. Thus, in order to clarify the broader factors related to housing solutions for this population, a systematic review was conducted to identify and synthesise the current research evidence (post-2003) and guide future housing design and development opportunities.
\end{abstract}

From the included studies ( $n=26), 198$ unique housing features were identified. From the 198 features, 142 related to housing design (i.e., internal or external characteristics of the dwelling and its land), 12 related to the dwelling's location (i.e., its proximity to available resources), and 54 related to the nature of the surrounding neighbourhood (i.e., the physical, social, and economic conditions of the area). The findings of this review contribute 
significantly to the literature by reporting a broader scope of relevant housing features for people with neurological disability, presenting preliminary guiding principles for housing design and development for this population, and identifying opportunities for future research. Keywords: complex disability; physical disability; cognitive disability; inclusive housing; supported accommodation; housing choice 


\section{What housing features should inform the development of housing solutions for adults with neurological disability?: A systematic review of the literature}

\section{Introduction}

Australians aged between 18 and 65 years ${ }^{1}$ with a complex disability who have high healthcare and support needs are at greatest risk of poor housing outcomes compared to mainstream society (Commonwealth of Australia, 2015). This population may include men and women with acquired or congenital conditions such as brain or spinal injury, multiple sclerosis, or cerebral palsy, who are in need of intensive personal care and support. Due to the physical, cognitive, sensory/perceptual, communicative, and/or behavioural consequences directly related to their neurological injury or illness (Hoppestad, 2006; MacDonald, 2016), individuals typically have limited or no choice regarding where they live, how they live, and with whom they live. A lack of housing options remains a contributing factor for many thousands of younger people who are often forced to live with ageing parents in the family home, or who are placed in group home or aged care residential settings with healthcare support (Commonwealth of Australia, 2015).

Despite the recent emphasis in Australian political, academic, and legis lative narratives to more actively promote real housing choice for people with high healthcare and

\footnotetext{
${ }^{1}$ After the age of 65 years, under current legislation, Australians become eligible for aged care accommodation services which includes residential care nursing homes and independent living facilities (Commonwealth of Australia, 2016). Support services, including funding for home modifications, for Australian adults with disability aged between 18 and 65 years will soon be provided under the National Disability Insurance Scheme (NDIS). The NDIS represents the most significant disability policy reform in Australian history and is progressively being rolled out across the country with full implementation expected by the end of 2019 (Wiesel \& Habibis, 2015). See the Australian Department of Human Services (n.d.) for more information about the NDIS.
} 
support needs, there is a lack of understanding regarding the specific housing features that might constitute better housing solutions for this population. Recognising the need for the housing development sector to provide more suitable housing options for people with disability (in addition to families with young children, individuals who sustain a temporary injury, and the elderly), a set of housing guidelines were developed by Livable Housing Australia (2012). The Livable Housing Design guidelines represent an important initiative that aims to increase the accessibility and visitability of private housing and specifies 16 housing design features (seven of which are considered a minimum standard) (Livable Housing Australia, 2012). The Livable Housing Design approach mirrors international strategies currently employed by Canada (e.g., the Accessibility for Ontarians Act [2005]; the FlexHousing concept as part of the universal design movement [Canada Mortgage and Housing Corporation, 2012]), the United States (e.g., the seven design and construction requirements of the Fair Housing Act for all new covered multifamily housing of more than four units [U.S. Department of Housing and Urban Development, 2016]), the United Kingdom (e.g., universal design Lifetime Homes standards in London; visitable standard of access for all new housing on a national scale [Scotts et al, 2007]), and Japan (e.g., who have a target of $40 \%$ of all housing stock to promote aging in place [Scotts et al., 2007]), to increase housing choice for people with disability. Australian disability advocates have championed the Livable Housing Design scheme, calling for the voluntary uptake of universal design to be mandated and regulated (Australian Network for Universal Housing Design and RI Australia, 2014).

Although inclusive (also referred to as supported or supportive) housing provision in Australia has rightly emphasised safety and accessibility features in the past, it has often failed to incorporate features related to broader psychosocial elements of housing such as dwelling location, neighbourhood quality, and overall design. Research has long confirmed 
the mutual relationship between place and people (Cummins et al., 2007) where physical and social elements of place (including and beyond physical accessibility) are key determinants of physical, psychological, and social (biopsychosocial) health and wellbeing (Carlson et al., 2012; Grant et al., 2014; Orrell et al., 2013). Social determinants of health models also clearly articulate the integration of housing and population health. The Social Determinants of Health and Environmental Health Promotion Framework (Schulz \& Northridge, 2004) confirms the importance of multiple factors within the broader built environment when considering the biopsychosocial health and wellbeing of individuals. In addition to the physical design of the dwelling, the housing location (i.e., its distance to available community-based resources), and the nature of the surrounding neighbourhood (i.e., physical, social, and economic conditions of the area influence the provision and adequacy of available resources) also affects the wellbeing of individuals by enhancing or hindering their access to resources (Hempel \& Tucker Jr., 1979; Lindberg et al., 1988; Schulz \& Northridge, 2004).

Despite the progressive views of governments, researchers, and housing providers relating to the need for contemporary inclusive housing solutions, and regardless of recent research and development initiatives in the housing sector recognising a vast array of housing design opportunities, there remains a lack of clarity regarding the broad set of housing features that combine to influence real housing solutions for people with complex disability. There is also a lack of understanding from the perspective of people with a disability regarding their own housing needs. A comprehensive understanding of housing features that should inform housing development, and are conducive to wellness, as reported from the 
consumers ${ }^{2}$ perspective for improved housing outcomes is needed.

This systematic review therefore aimed to identify and synthesise the current research evidence relating to all housing features that could inform housing design and development decisions for adults between 18 years and 65 years of age with neurological disability. The search timeframe was set according to the introduction of a government-led disability services framework in Australia that promoted inclusive communities for people with disability and their carers. The Third Commonwealth State/Territory Disability Agreement (CSTDA; Commonwealth of Australia, 2003) was based on the principle that "communities are enriched by the inclusion of people with disabilities and that positive assumptions about the gifts and capacities of people with disabilities, including those with high support needs, are fundamental to their experience of a good life” (p. 2). Thus, the agreement was a milestone regarding the inclusion of people with disability and heralded a major shift in how public, private, and philanthropic organisations considered future housing for people with disability. Given the focus of the Third CSTDA (Commonwealth of Australia, 2003) on purposefully promoting the inclusion of people with disability in the community, this systematic review sought relevant literature published after 01 January 2003 (i.e., post-2003). Further, in accordance with the social determinants of health promotion framework (Schultz \& Northridge, 2004), it was also important to contemplate residential environments within the broader psychosocial context. Although some scholars have combined location and neighbourhood features into a single category (Krieger \& Higgins 2002; Megbolugbe et al. 1991), others have preserved them as separate entities (Kauko 2006; Lindberg et al. 1988;

\footnotetext{
${ }^{2}$ Since the person's family and non-family paid carers are often involved in decisions regarding where the person with disability will live, with or on behalf of the individual (as guardian or power of attorney), and/or providing care and support for the person, thereby also interacting with the residential environment (Saugeres, 2011), the term 'consumer' is used throughout to refer to adults with neurological disability and their family/non-family carers.
} 
Shaw 2004). For the purpose of this article, location relates to 'distance' (i.e., proximity to available resources) and neighbourhood refers to ‘the provision of adequate resources' (i.e., the physical, social, and economic conditions of the area). Thus, location and neighbourhood housing features are described as forming two separate housing domains.

Given the absence of an agreed framework from which to understand key housing features for people with neurological disability, combined with the evidence that the physical design of housing, where housing is located, and the nature of the surrounding neighbourhood are central to a person's biopsychosocial health and wellbeing (Hempel \& Tucker Jr., 1979; Lindberg et al., 1988; Schulz \& Northridge, 2004), this review sought to examine the housing features that, at least, reflect these important domains of housing design, location, and neighbourhood. To address the research aim, the following research question was proposed for this systematic review:

What housing features (inclusive of design, location, and neighbourhood) reported in the literature ought to (or should not) inform housing design and development for adults with neurological disability?

\section{Method}

The review protocol described below was registered a priori on the PROSPERO database (http^//www.crd.york.ac.uk/PROSPERO/) under registration number CRD42014010751. The systematic review methodology and findings are reported according to the PRISMA reporting guidelines (Moher et al., 2009).

\section{Search strategy}

The initial search of the literature was conducted between the $6^{\text {th }}$ and $12^{\text {th }}$ of April, 2014. A search for scientific and grey literature was undertaken to broaden the scope of potentially relevant studies that might answer the research question, and in doing so, limit publication bias that might affect the results (Mahood et al., 2014). An updated search of the literature 
was undertaken in April 2016 to capture recently published articles that met the eligibility criteria. The information reported below represents the cumulative (01 January 2003 - 30 April 2016) method and review findings.

\section{Electronic databases: Scientific and grey literature}

Four electronic databases were each searched for published peer-reviewed articles and grey literature, as available: Avery Index to Architectural Periodicals, CINAHL, PubMed/Medline, and ProQuest Social Science Journals. Combinations of search terms relating to (a) housing type, (b) the target population, (c) preferences and perspectives, (d) housing features, and (e) exclusionary keywords based on the exclusion criteria (described on page 10) resulted in a search matrix of 126 search combinations (see Appendix A). The search matrix was then applied to each electronic database (see Appendices B, C, D, and E, respectively) where keyword search terms were replaced with subject headings indexed to each database to increase the number of relevant studies identified (Bell, 2012). Limiters including language (English), publication date (01 January 2003 - 30 April 2016), human rather than animal (i.e., CINAHL and PubMed / Medline databases), and publication type (scientific literature or grey literature, respectively) were also set. The limiters specified conformed to the review inclusion and exclusion criteria.

\section{Other sources: Grey literature}

Given the recent political, academic, and legislative emphasis in Australia to more actively promote consumer housing choice, the Australian Institute of Health and Welfare (AIHW) website, the Australian Housing and Urban Research Institute (AHURI) website, and the Summer Foundation website were systematically searched for relevant grey literature. Although exclusionary keywords and subject headings could not be used, the search strategy remained "systematic, transparent and reproducible” (Mahood et al., p. 221). For example, rather than using a search matrix (as was done to systematically search each electronic 
database), only the term "disability" was entered into the search bar for the two websites with large and complex datasets: the AIHW (health and welfare focus) and AHURI (housing focus) websites. Given the specific focus of the Summer Foundation on housing for people with disability, the search bar was not used to retrieve records when searching this website. All returned results (AIHW and AHURI websites) and all publications listed (Summer Foundation website) between 01 January 2003 and 30 April 2016 were identified for further screening.

\section{Eligibility criteria}

\section{Inclusion criteria}

Studies were included in the review if they met each of the following criteria:

1. Comprised empirical (human-based) research;

2. Published between 01 January 2003 and 30 April 2016;

3. Written in English;

4. Primarily related to community-based housing for adults aged 18-64 years with a principal neurological diagnosis (i.e., damage to the central nervous system was the primary disability) of sufficient severity or complexity that the person would likely require some form of informal or paid care or support;

5. Reported on features relating to (a) housing design (i.e., internal and external characteristics of the dwelling and its land); and/or (b) location (i.e., proximity to available resources); and/or (c) neighbourhood (i.e., the physical, social, and economic conditions of the area), in line with the broad housing classification framework originally proposed by Hempel and Tucker Jr. (1979) and confirmed by Lindberg and colleagues (1988); 
6. Reported results (a) from the perspective of adults aged 18-64 years with a principal neurological diagnosis (Sample 1) and/or their family/non-family carers (Sample 2); or (b) experimental evidence relating to adults with neurological disability;

7. Reported results that described housing features in some way (e.g., viewed positively or negatively) so that meaningful conclusions could be drawn; and

8. Presented original data. To ensure no overrepresentation of a particular set of data, two screening approaches were used: (1) for separate studies that used the same dataset (i.e., a preliminary report and a final report), only the paper with the most detailed reporting was included; and (2) separate studies conducted by the same researchers that collected different data but aimed to address the same overall research questions were combined to represent only one study.

\section{Exclusion criteria}

Articles were excluded if they did not meet the above criteria, or more specifically: (a) primarily focused on the housing needs of individuals with a principal mental health diagnosis, intellectual disability or developmental disability (otherwise not defined as neurological/cognitive impairment), or individuals diagnosed with Dementia or Alzheimer’s disease given that these conditions are particularly prevalent amongst the elderly (i.e., aged 65 years and over) (Alzheimer's Association, 2015; World Health Organization [WHO], 2012); (b) encompassed a clinical focus rather than a housing focus (e.g., reported medical intervention outcomes, rehabilitation/exercise techniques, or recovery programs not related to housing design or development); (c) represented a stakeholder perspective (e.g., medical doctors) rather than a consumer perspective (e.g., people with disability; family/non-family carers); (d) did not meet the specified age criteria (18-64 years); and (e) focused on relational factors such as staff-client relationships/staff qualities rather than housing design (i.e., internal and external characteristics of the dwelling and its surrounds). 


\section{Screening and study selection}

Records were screened to remove duplicate studies by entering them into software-based reference management system, Endnote $($ (Thomson Reuters, 2014, v 17.1.0.7705). After removing the duplicates, each title and abstract (scholarly literature) or executive summary (grey literature, where available) were evaluated for eligibility. Full-text articles were retrieved when eligibility could not be determined from the abstract alone.

\section{Data extraction and synthesis}

A standard data extraction form was used to provide consistency and structure to the data extraction process. Data extraction was performed independently by two researchers to limit selection bias. Any differences were reconciled by mutual agreement with a third researcher. A narrative synthesis was used to describe the evidence obtained from the included studies.

\section{Quality appraisal}

Two researchers independently assessed the methodological rigor of all included studies (Researcher 1) and a subsample (50\%) of the included studies (Researcher 2), using the Mixed-Methods Appraisal Tool (MMAT; Pace et al., 2012; Pluye et al., 2011). The assessed studies were given an overall quality score (expressed as a percentage) by each reviewer. Inter-rater agreement was calculated and indicated substantial agreement $(\kappa=0.763 ; p<0.001)$ between the researchers (Landis \& Koch, 1977). Minor discrepancies in scoring were resolved through discussion with a third researcher.

\section{Results}

Figure 1 outlines the review process undertaken according to the PRISMA 2009 Flow Diagram guidelines (Moher et al., 2009). Twenty-two eligible studies (i.e., 15 journal articles; five reports; two dissertations) were initially identified. However, two reports that collected and analysed data to answer the same research aims/questions for two separate Australian states were combined, resulting in the identification of 21 eligible studies (i.e., 15 
journal articles; four reports; two dissertations) in total. As per the review protocol, the reference lists of the 21 eligible studies were then hand searched for additional articles that satisfied the inclusionary criteria. Five additional journal articles were subsequently identified, resulting in the final inclusion of 26 studies (i.e., 20 journal articles; four reports; two dissertations) in the review. Data from the final 26 included studies were extracted for synthesis. 


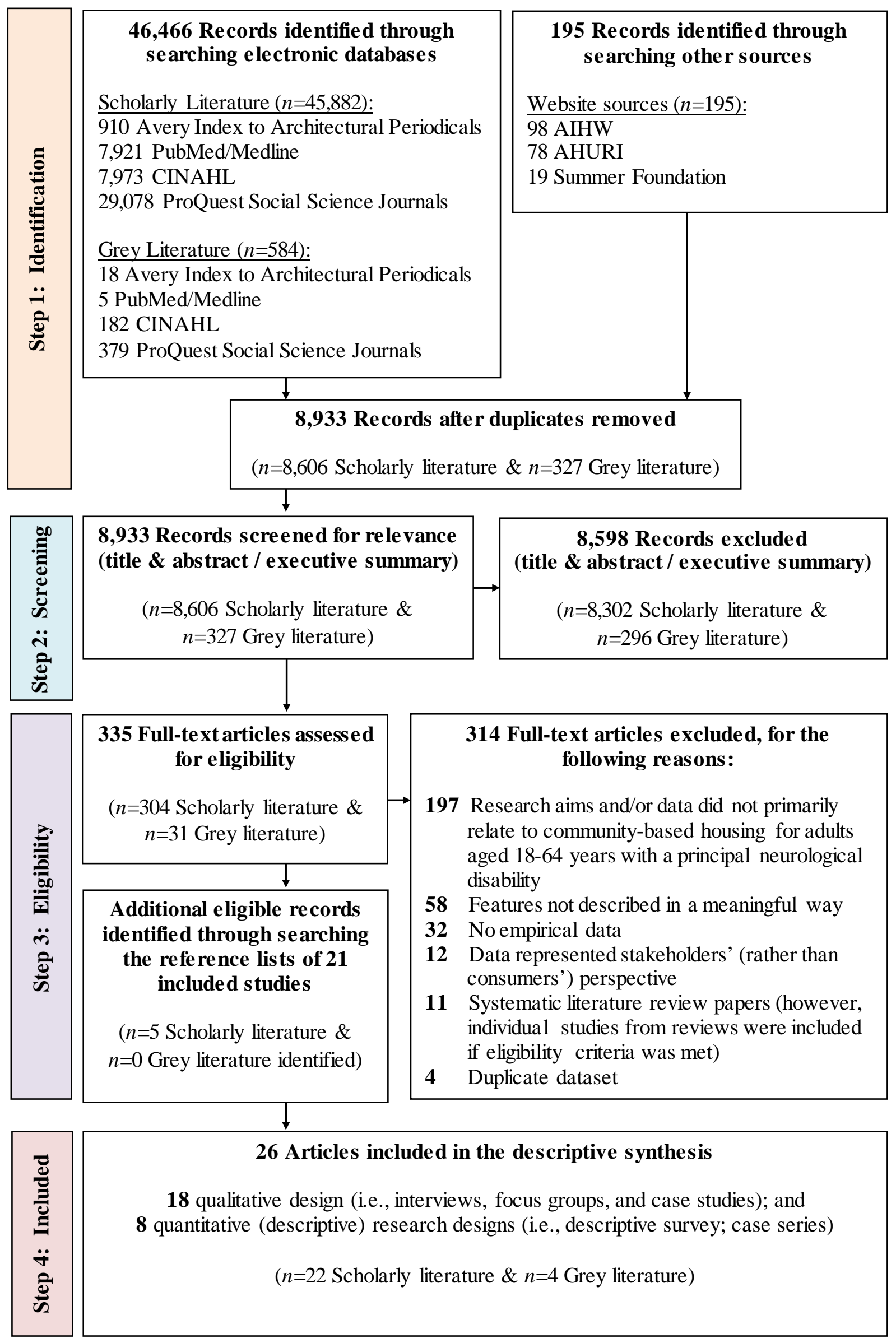

Figure 1. PRISMA 2009 Flow Diagram (adapted from Moher et al., 2009). 


\section{Study quality}

Final quality assessment scores for each included study are reported in Table 1. Despite the expression of an overall quality score (percentage) for all included studies, no assessment score 'cut-off' or score range indicating 'high quality', 'moderate quality', or 'low quality' studies have been empirically developed and tested for the MMAT (Pace et al., 2012; Pluye et al., 2011). Notwithstanding the lower scorings in some domains, all 26 (100\%) included studies satisfied internal and external validity (Higgins et al., 2011). Thus, no articles were excluded on the basis of quality appraisal. 
Table 1

Methodological Quality Assessment Scores for the 26 Included Studies

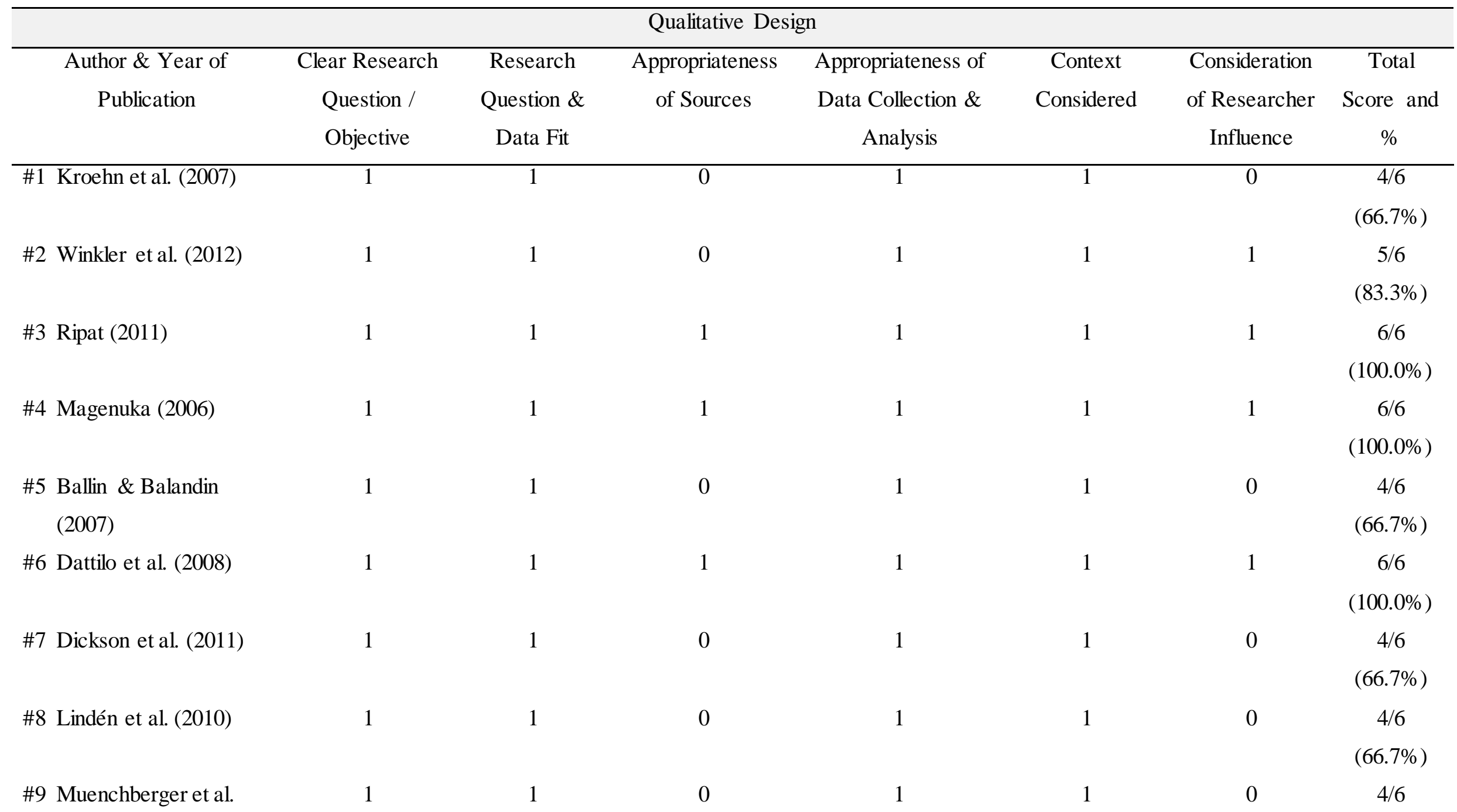




\begin{tabular}{|c|c|c|c|c|c|c|c|}
\hline \multicolumn{8}{|c|}{ Qualitative Design } \\
\hline $\begin{array}{l}\text { Author \& Year of } \\
\text { Publication }\end{array}$ & $\begin{array}{c}\text { Clear Research } \\
\text { Question / } \\
\text { Objective }\end{array}$ & $\begin{array}{c}\text { Research } \\
\text { Question \& } \\
\text { Data Fit }\end{array}$ & $\begin{array}{c}\text { Appropriateness } \\
\text { of Sources }\end{array}$ & $\begin{array}{c}\text { Appropriateness of } \\
\text { Data Collection \& } \\
\text { Analysis }\end{array}$ & $\begin{array}{c}\text { Context } \\
\text { Considered }\end{array}$ & $\begin{array}{c}\text { Consideration } \\
\text { of Researcher } \\
\text { Influence }\end{array}$ & $\begin{array}{c}\text { Total } \\
\text { Score and } \\
\%\end{array}$ \\
\hline (2012) & & & & & & & $(66.7 \%)$ \\
\hline \#10 Yeung et al. (2008) & 1 & 1 & 1 & 1 & 1 & 1 & $\begin{array}{c}6 / 6 \\
(100.0 \%)\end{array}$ \\
\hline \#12 Reid et al. (2003) & 1 & 1 & 1 & 1 & 1 & 0 & $\begin{array}{c}5 / 6 \\
(83.3 \%)\end{array}$ \\
\hline $\begin{array}{l}\text { \#13 Pettersson et al. } \\
\text { (2014) }\end{array}$ & 1 & 1 & 0 & 1 & 1 & 0 & $\begin{array}{c}4 / 6 \\
(66.7 \%)\end{array}$ \\
\hline $\begin{array}{l}\text { \#15 Bozzolini \& } \\
\text { Cassibba (2008) }\end{array}$ & 1 & 1 & 1 & 1 & 1 & 0 & $\begin{array}{c}5 / 6 \\
(83.3 \%)\end{array}$ \\
\hline $\begin{array}{l}\text { \#16 Linskell \& } \\
\text { Bouamrane (2012) }\end{array}$ & 1 & 1 & 1 & 1 & 1 & 0 & $\begin{array}{c}5 / 6 \\
(83.3 \%)\end{array}$ \\
\hline $\begin{array}{l}\text { \#17 Pettersson et al. } \\
\text { (2012) }\end{array}$ & 1 & 1 & 1 & 1 & 1 & 1 & $\begin{array}{c}6 / 6 \\
(100.0 \%)\end{array}$ \\
\hline \#18 Sakellariou (2015) & 1 & 1 & 1 & 1 & 1 & 0 & $\begin{array}{c}5 / 6 \\
(83.3 \%)\end{array}$ \\
\hline
\end{tabular}




\begin{tabular}{|c|c|c|c|c|c|c|c|}
\hline \multicolumn{8}{|c|}{ Qualitative Design } \\
\hline $\begin{array}{l}\text { Author \& Year of } \\
\text { Publication }\end{array}$ & $\begin{array}{l}\text { Clear Research } \\
\text { Question / } \\
\text { Objective }\end{array}$ & $\begin{array}{c}\text { Research } \\
\text { Question \& } \\
\text { Data Fit }\end{array}$ & $\begin{array}{l}\text { Appropriateness } \\
\text { of Sources }\end{array}$ & $\begin{array}{l}\text { Appropriateness of } \\
\text { Data Collection \& } \\
\text { Analysis }\end{array}$ & $\begin{array}{c}\text { Context } \\
\text { Considered }\end{array}$ & $\begin{array}{l}\text { Consideration } \\
\text { of Researcher } \\
\text { Influence }\end{array}$ & $\begin{array}{c}\text { Total } \\
\text { Score and } \\
\%\end{array}$ \\
\hline Total Sum & $\begin{array}{c}18 \\
\text { (out of 18) }\end{array}$ & $\begin{array}{c}18 \\
\text { (out of 18) }\end{array}$ & $\begin{array}{c}9 \\
\text { (out of 18) }\end{array}$ & $\begin{array}{c}18 \\
\text { (out of } 18 \text { ) }\end{array}$ & $\begin{array}{c}16 \\
\text { (out of } 18 \text { ) }\end{array}$ & $\begin{array}{c}6 \\
\text { (out of 18) }\end{array}$ & \\
\hline $\begin{array}{c}\text { Author \& Year of } \\
\text { Publication }\end{array}$ & $\begin{array}{l}\text { Clear Research } \\
\text { Question / } \\
\text { Objective }\end{array}$ & $\begin{array}{c}\text { Research } \\
\text { Question \& } \\
\text { Data Fit }\end{array}$ & $\begin{array}{l}\text { Sampling } \\
\text { Strategy }\end{array}$ & $\begin{array}{c}\text { Sample } \\
\text { Representativeness }\end{array}$ & $\begin{array}{c}\text { Appropriateness } \\
\text { of Measures }\end{array}$ & $\begin{array}{c}\text { Acceptable } \\
\text { Response Rate }\end{array}$ & $\begin{array}{c}\text { Total } \\
\text { Score and } \\
\%\end{array}$ \\
\hline $\begin{array}{l}\text { \#19 Winkler et al. (2007) } \\
\text { \& Winkler et al. } \\
\text { (2008) }\end{array}$ & 1 & 1 & 0 & 1 & 1 & 0 & $\begin{array}{c}4 / 6 \\
(66.7 \%)\end{array}$ \\
\hline $\begin{array}{l}\text { \#21 Pettersson et al. } \\
\text { (2015) }\end{array}$ & 1 & 1 & 0 & 1 & 1 & 0 & $\begin{array}{c}4 / 6 \\
(66.7 \%)\end{array}$ \\
\hline \#22 Dutta et al. (2011) & 1 & 1 & 1 & 0 & 1 & N/A & $\begin{array}{c}4 / 5 \\
(80.0 \%)\end{array}$ \\
\hline \#23 Koontz et al. (2005) & 1 & 1 & 0 & 0 & 1 & N/A & $\begin{array}{c}3 / 5 \\
(60.0 \%)\end{array}$ \\
\hline \#24 Koontz et al. (2010) & 1 & 1 & 0 & 0 & 1 & N/A & $3 / 5$ \\
\hline
\end{tabular}




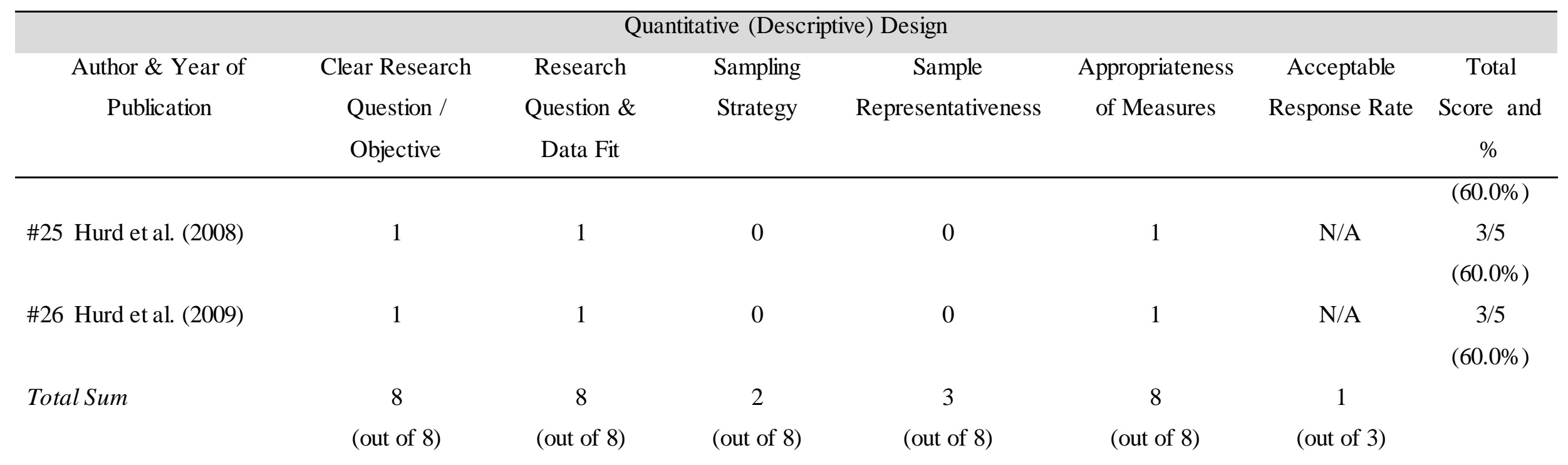

Note. Coding: 1 = yes; $0=$ no/not sure (not mentioned / not mentioned in sufficient detail). 


\section{Description of included studies}

The 26 included studies were published between 2003 and 2015. Twenty-four of the 26 studies (92\%) described housing design features, seven studies (27\%) described location features, and 17 of the 26 studies (65\%) described neighbourhood features relevant to adults with neurological disability. Twenty-one of the 26 studies (81\%) presented the perspective of consumers (i.e., adults with neurological disability; family/non-family carers) and the five remaining studies (19\%) presented observational evidence using a case series design. Of the 21 studies that focused on consumer perspectives, all 21 included the voice of adults with neurological disability. Five of these 21 studies also included the views of the person's family/non-family carers. However, one of these five studies (\#11) included non-family paid staff yet housing features identified by these participants were not reported. Another study (\#19) did not report the views of individuals with disability and carers separately so the findings were deemed to relate to both samples.

The total reported sample size was 684 (two studies did not specify the number of family and/or non-family carers that participated in the research). Individual study sample sizes ranged from one person (case study design) to 223 individuals (case series design). The reporting of age range and gender for adults with neurological disability and family/nonfamily carers was inconsistent across the studies, so could not be accurately calculated. For a more detailed description of the sample (and sub-samples) of this review, see Appendix F. Table 2 presents an overview of the included studies. 
Table 2

Summary Table for 26 Studies Included in the Systematic Review

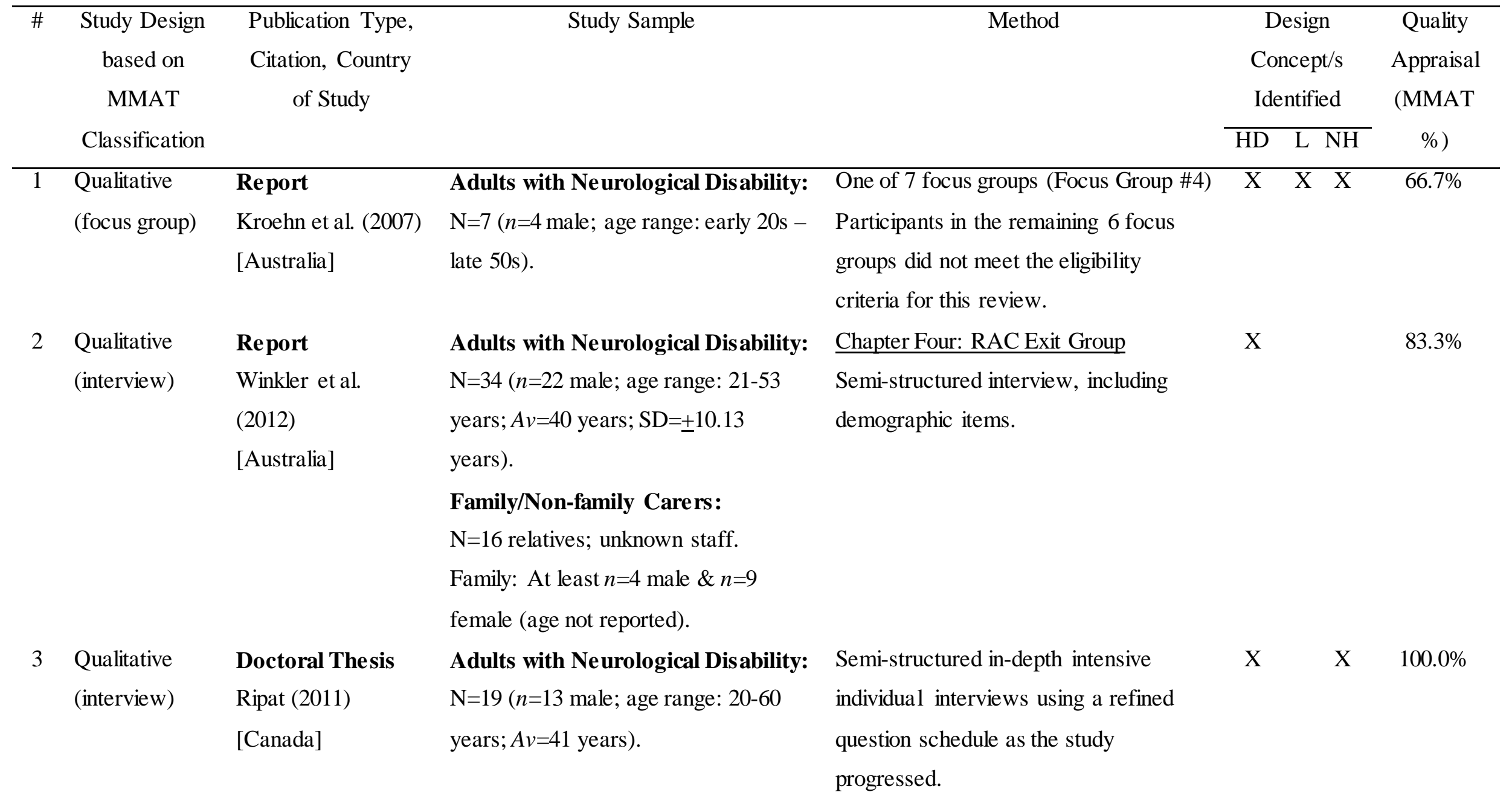




\begin{tabular}{|c|c|c|c|c|c|c|c|c|}
\hline \multirow[t]{2}{*}{ \# } & \multirow{2}{*}{$\begin{array}{l}\text { Study Design } \\
\text { based on } \\
\text { MMAT } \\
\text { Classification }\end{array}$} & \multirow[t]{2}{*}{$\begin{array}{l}\text { Publication Type, } \\
\text { Citation, Country } \\
\text { of Study }\end{array}$} & \multirow[t]{2}{*}{ Study Sample } & \multirow[t]{2}{*}{ Method } & \multicolumn{3}{|c|}{$\begin{array}{c}\text { Design } \\
\text { Concept/s } \\
\text { Identified }\end{array}$} & \multirow{2}{*}{$\begin{array}{l}\text { Quality } \\
\text { Appraisal } \\
\text { (MMAT } \\
\% \text { ) }\end{array}$} \\
\hline & & & & & $\mathrm{HD}$ & $\mathrm{L}$ & $\mathrm{NH}$ & \\
\hline 4 & $\begin{array}{l}\text { Qualitative } \\
\text { (interview) }\end{array}$ & $\begin{array}{l}\text { Doctoral Thesis } \\
\text { Magenuka (2006) } \\
\text { [South Africa] }\end{array}$ & $\begin{array}{l}\text { Adults with Ne urological Disability: } \\
\mathrm{N}=10 \text { ( } n=10 \text { male; age range: } 25-63 \\
\text { years). }\end{array}$ & Individual interviews. & $\bar{X}$ & & $\mathrm{X}$ & $100.0 \%$ \\
\hline 5 & $\begin{array}{l}\text { Qualitative } \\
\text { (interview) }\end{array}$ & $\begin{array}{l}\text { Journal Article } \\
\text { Ballin \& Balandin } \\
\text { (2007) } \\
\text { [Australia] }\end{array}$ & $\begin{array}{l}\text { Adults with Ne urological Disability: } \\
\mathrm{N}=7 \text { ( } n=4 \text { male; age range: } 40-64 \\
\text { years). }\end{array}$ & $\begin{array}{l}\text { In-depth interviews (each participant } \\
\text { interviewed twice). }\end{array}$ & & & $\mathrm{X}$ & $66.7 \%$ \\
\hline 6 & $\begin{array}{l}\text { Qualitative } \\
\text { (focus group) }\end{array}$ & $\begin{array}{l}\text { Journal Article } \\
\text { Dattilo et al. (2008) } \\
\text { [USA and Canada] }\end{array}$ & $\begin{array}{l}\text { Adults with Neurological Disability: } \\
\mathrm{N}=8 \text { ( } n=4 \text { male; age range: } 27-44 \\
\text { years). }\end{array}$ & $\begin{array}{l}\text { Focus group conducted via an online } \\
\text { forum. }\end{array}$ & $\mathrm{X}$ & $\mathrm{X}$ & $\mathrm{X}$ & $100.0 \%$ \\
\hline 7 & $\begin{array}{l}\text { Qualitative } \\
\text { (interview) }\end{array}$ & $\begin{array}{l}\text { Journal Article } \\
\text { Dickson et al. } \\
\text { (2011) } \\
{[\mathrm{UK}]}\end{array}$ & $\begin{array}{l}\text { Adults with Neurological Disability: } \\
\mathrm{N}=17 \text { ( } n=14 \text { male; age range: } 26-62 \\
\text { years; } A v=46 \text { years). }\end{array}$ & Individual semi-structured interviews. & $\mathrm{X}$ & & $\mathrm{X}$ & $66.7 \%$ \\
\hline 8 & $\begin{array}{l}\text { Qualitative } \\
\text { (interview) }\end{array}$ & $\begin{array}{l}\text { Journal Article } \\
\text { Lindén et al. (2010) } \\
\text { [Sweden] }\end{array}$ & $\begin{array}{l}\text { Adults with Neurological Disability: } \\
\mathrm{N}=36 \text { ( } n=27 \text { male; age range: } 26-60 \\
\text { years; } A v=44 \text { years). }\end{array}$ & $\begin{array}{l}\text { Descriptive interview study using a } \\
\text { revised and extended version of the } \\
\text { ETUQ (a semi-structured standardized } \\
\text { interview questionnaire; } 104 \text { items). }\end{array}$ & $\mathrm{X}$ & & $\mathrm{X}$ & $66.7 \%$ \\
\hline
\end{tabular}




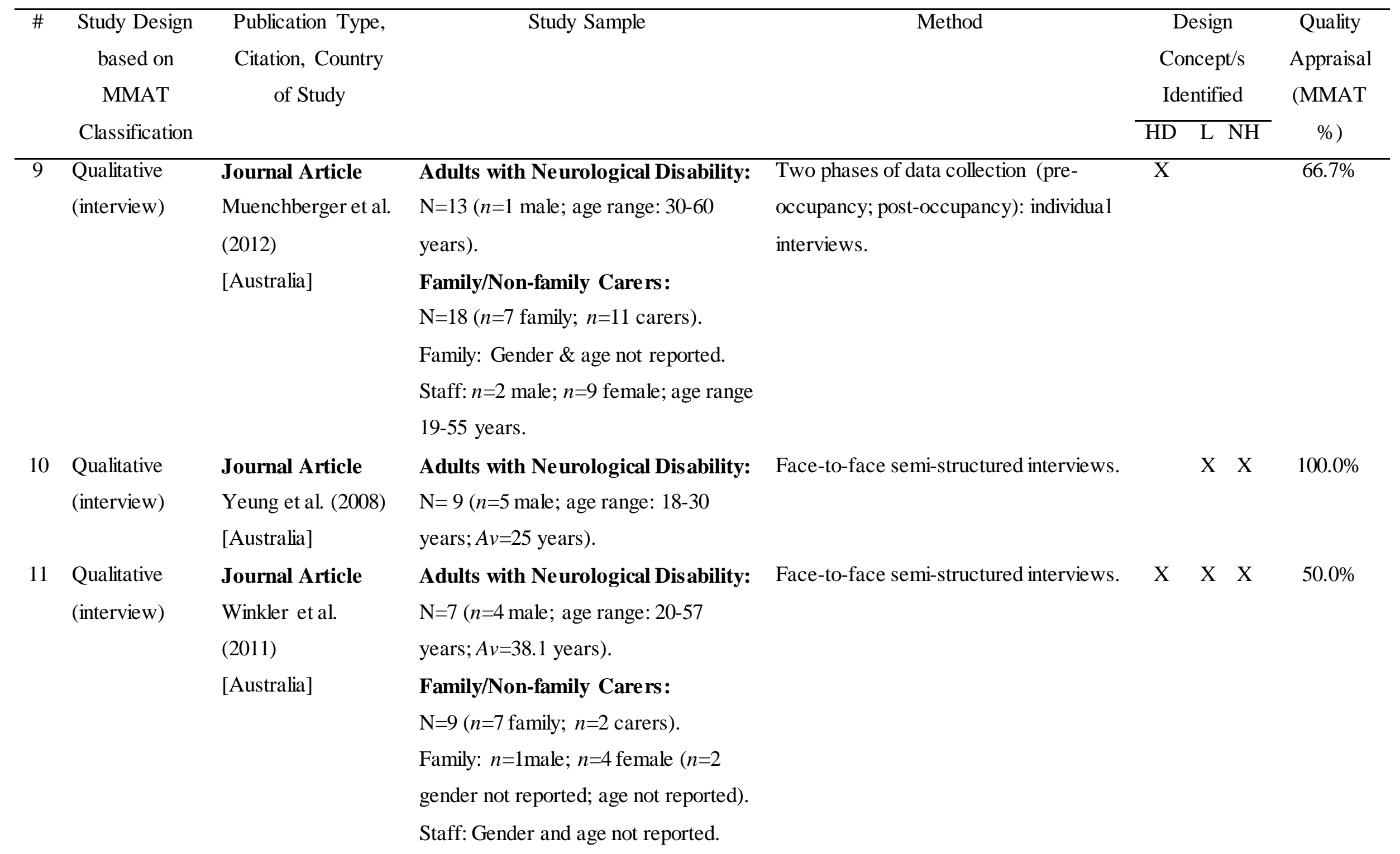




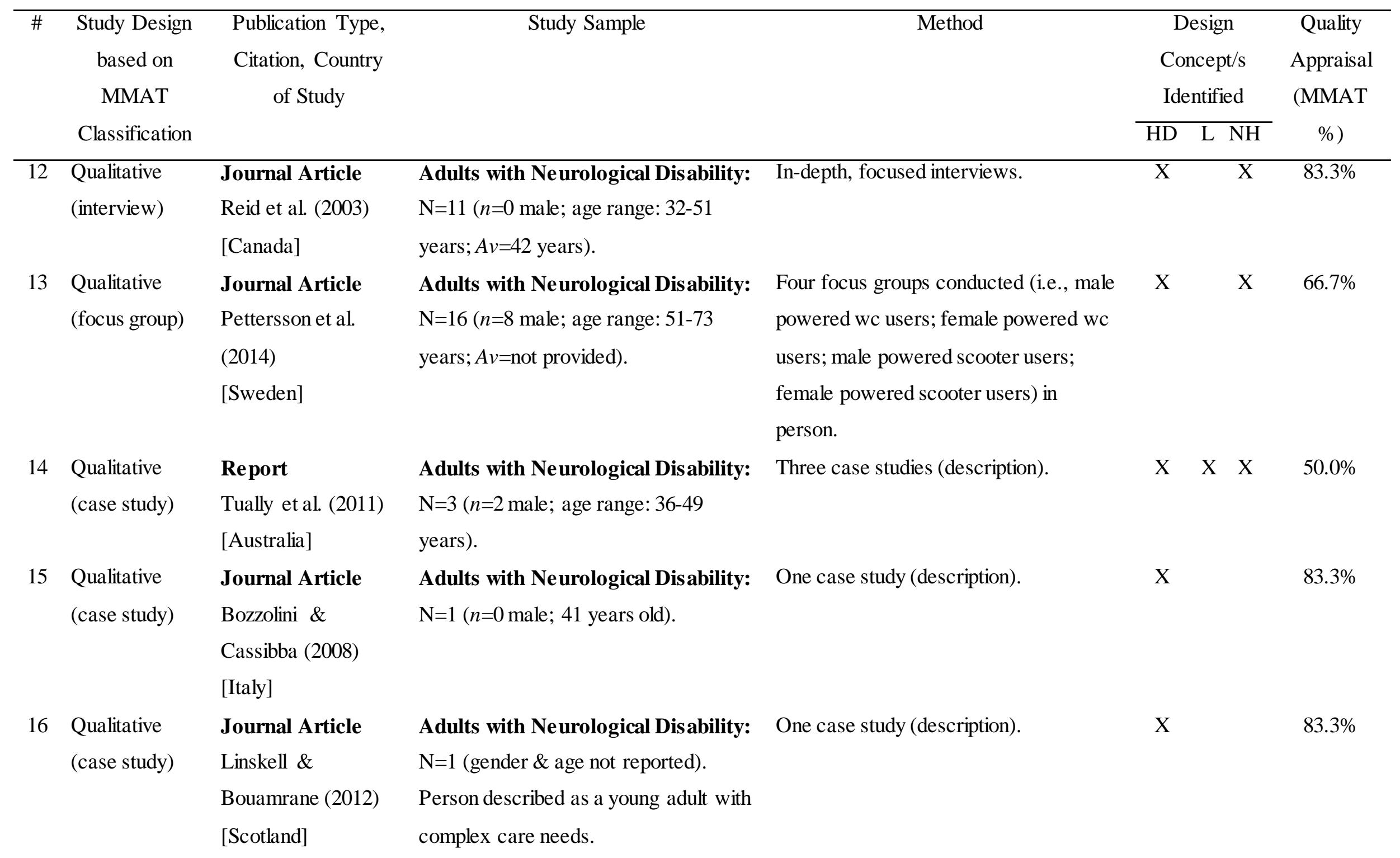




\begin{tabular}{|c|c|c|c|c|c|c|c|}
\hline \multirow[t]{2}{*}{$\#$} & \multirow{2}{*}{$\begin{array}{c}\text { Study Design } \\
\text { based on } \\
\text { MMAT } \\
\text { Classification }\end{array}$} & \multirow[t]{2}{*}{$\begin{array}{l}\text { Publication Type, } \\
\text { Citation, Country } \\
\text { of Study }\end{array}$} & \multirow[t]{2}{*}{ Study Sample } & \multirow[t]{2}{*}{ Method } & \multicolumn{2}{|c|}{$\begin{array}{c}\text { Design } \\
\text { Concept/s } \\
\text { Identified }\end{array}$} & \multirow{2}{*}{$\begin{array}{c}\text { Quality } \\
\text { Appraisal } \\
\text { (MMAT } \\
\% \text { ) }\end{array}$} \\
\hline & & & & & HD & $\mathrm{L} \mathrm{NH}$ & \\
\hline 17 & $\begin{array}{l}\text { Qualitative } \\
\text { (case study) }\end{array}$ & $\begin{array}{l}\text { Journal Article } \\
\text { Pettersson et al. } \\
\text { (2012) } \\
\text { [Sweden] }\end{array}$ & $\begin{array}{l}\text { Adults with Ne urological Dis ability: } \\
\mathrm{N}=3 \text { ( } n=1 \text { male; age range: } 39-59 \\
\text { years). }\end{array}$ & $\begin{array}{l}\text { Three longitudinal case studies } \\
\text { (description). }\end{array}$ & $\bar{X}$ & $\mathrm{X}$ & $100.0 \%$ \\
\hline 18 & $\begin{array}{l}\text { Qualitative } \\
\text { (case study) }\end{array}$ & $\begin{array}{l}\text { Journal Article } \\
\text { Sakellariou (2015) } \\
{[\mathrm{UK}]}\end{array}$ & $\begin{array}{l}\text { Adults with Neurological Dis ability: } \\
\mathrm{N}=1 \text { ( } n=1 \text { male; age: “late } 50 \text { 's”). } \\
\text { Family/Non-family Carers: } \\
\mathrm{N}=1 \text { ( } n=1 \text { family; } n=0 \text { carers). } \\
\text { Family: } n=0 \text { male; age: “late } 50 \text { 's”). }\end{array}$ & One case study (description). & $\mathrm{X}$ & & $83.3 \%$ \\
\hline 19 & $\begin{array}{l}\text { Quantitative } \\
\text { Descriptive } \\
\text { Design } \\
\text { (survey) }\end{array}$ & $\begin{array}{l}\text { Report } \\
\text { Winkler et al. } \\
\text { (2007) } \\
\text { [Australia] }\end{array}$ & $\begin{array}{l}\text { Adults with Neurological Disability: } \\
\mathrm{N}=105 \text { ( } n=61 \text { male; age range: } 20-50 \\
\text { years; } A v=42 \text { years). Three sub-groups } \\
\text { of participants with similar levels of } \\
\text { support needs identified (i.e., 'very } \\
\text { high care needs' group [N=52; } n=28 \\
\text { male]; 'high care needs' group [N=35; } \\
n=18 \text { male]; and 'moderate care needs' } \\
\text { group [N=16; } n=14 \text { male]). }\end{array}$ & $\begin{array}{l}\text { Participants: The first } 105 \text { individuals } \\
\text { aged under } 50 \text { years living in RAC in } \\
\text { Victoria who underwent the my future } \\
\text { my choice assessment and planning } \\
\text { process. }\end{array}$ & & $\mathrm{X} \quad \mathrm{X}$ & $66.7 \%$ \\
\hline
\end{tabular}




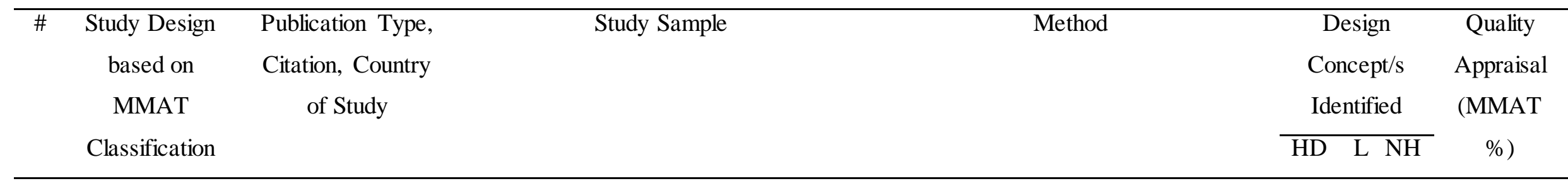

Family/Non-family Carers:

$\mathrm{N}=$ not reported

$\begin{array}{ll}\text { Quantitative } & \text { Report } \\ \text { Descriptive } & \text { Winkler et al. } \\ \text { Design } & \text { (2008) } \\ \text { (survey) } & \text { [Australia] }\end{array}$

$\mathrm{N}=14$ ( $n=7$ male; age range: $34-50$

years; $A v=45$ years).

Family/Non-family Carers:

$\mathrm{N}=$ not reported

20 Quantitative

Descriptive

Design

(survey)

\section{Journal Article}

Boman et al. (2007)

[Sweden]

\section{Nenteported}

Adults with Neurological Disability: Participants: 14 of the 16 individuals

my

Participants: 14 of the 16 individuals
aged under 50 years living in RAC in
Tasmania who underwent the my future

my choice assessment and planning

process.

The same dimensions assessed in the

Victorian study were assessed in this

study, using the same measures.

Adults with Neurological Disability: Two study apartments (1x one-bedroom

$\mathrm{X}$

$100.0 \%$

apartment for one person; and $1 \mathrm{x}$ two-

bedroom apartment for a couple) were

equipped with basic and advanced

EADL. Participants were taught to use

all EADL and stayed in the apartments

for four $(n=4)$ or six months $(n=4)$.

Function and QOL were assessed with 


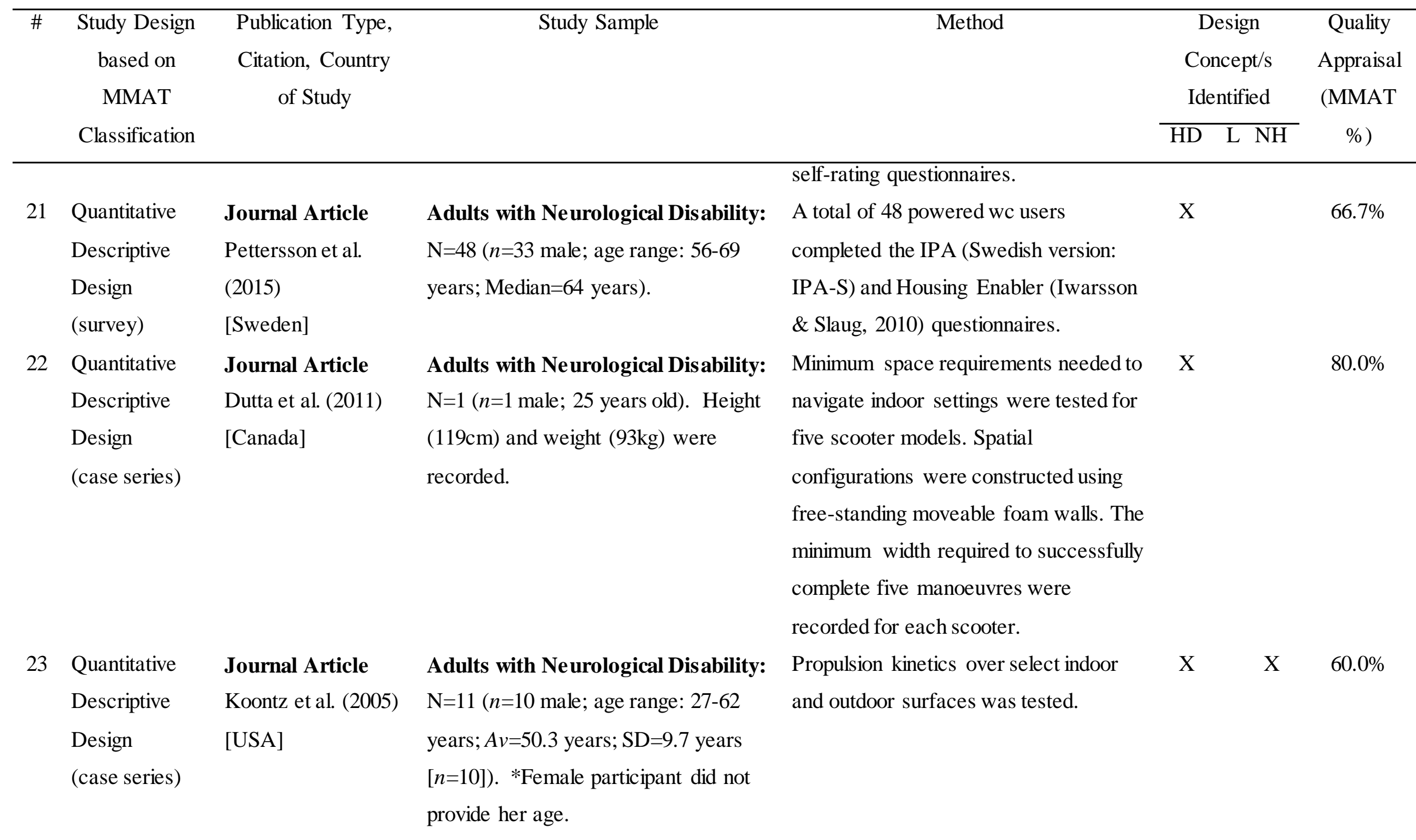




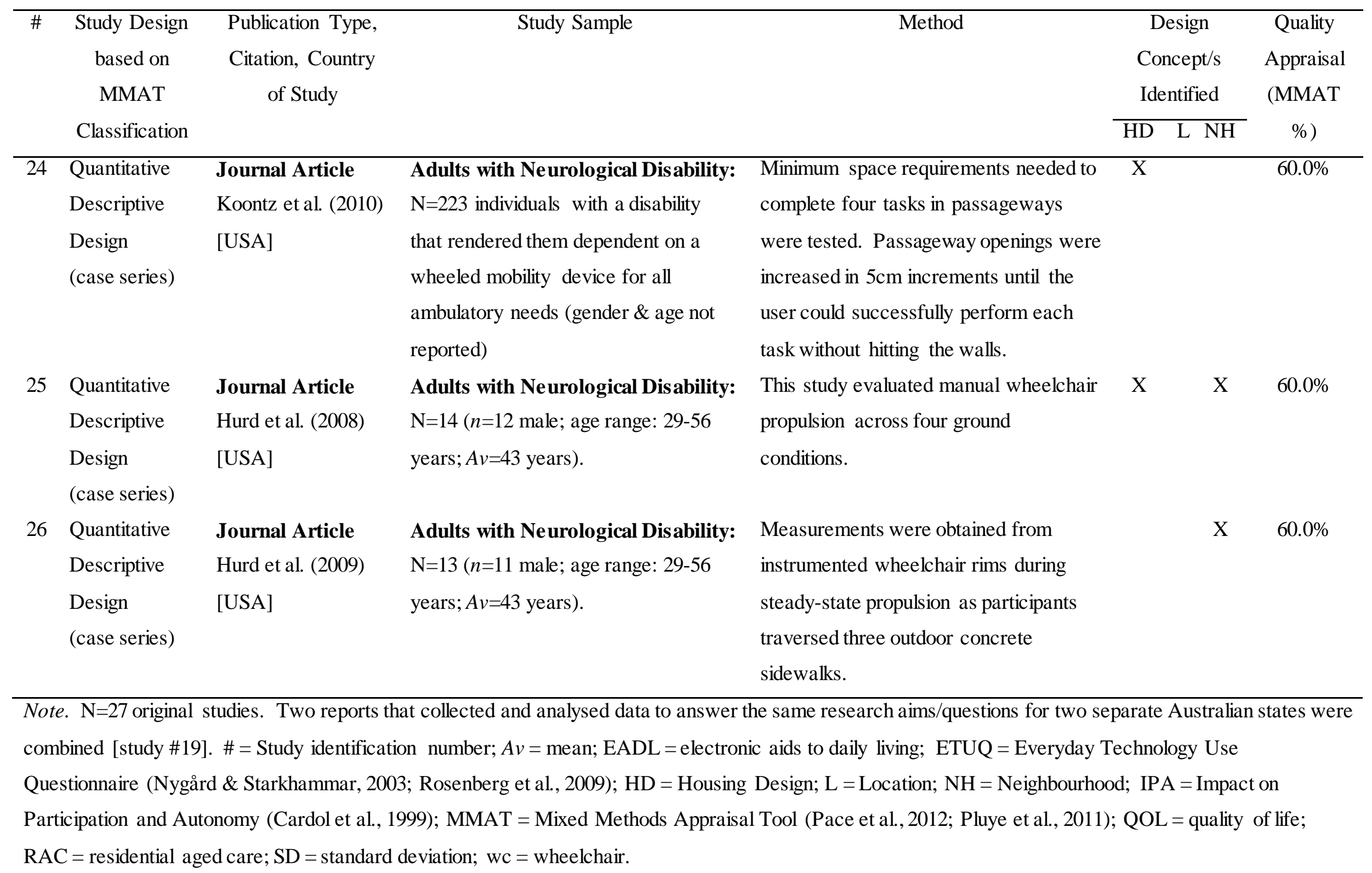




\section{Housing features identified}

A comprehensive set of housing features relating to housing design, location, and neighbourhood were identified. From the included studies ( $n=26), 198$ unique housing features were identified (see Appendix G for a full list of features, and the studies and population group/s that identified them). From the 198 features, 142 related to housing design (i.e., internal or external characteristics of the dwelling and its land), 12 related to the dwelling's location (i.e., its proximity to available resources), and 54 related to the nature of the surrounding neighbourhood (i.e., the physical, social, and economic conditions of the area). Interestingly, 10 features relating to floor/ground surfaces, minimum space requirements to manoeuvre through passageways, and automated doors were associated with housing design and the neighbourhood context.

\section{Housing design}

The 142 housing design features reported in the included studies related to six key themes:

(1) physical access; (2) a 'homely' environment; (3) resident safety and security; (4) privacy;

(5) supportive care features; and (6) rehabilitation and exercise. Table 3 presents an overview of the results. 
Table 3

Number and Percentage of Housing Design Features Reported by the 26 Included Studies

\begin{tabular}{|c|c|c|c|c|c|c|c|c|}
\hline \multirow[t]{2}{*}{ Theme } & \multicolumn{8}{|c|}{$\begin{array}{l}\text { Housing Design Features Identified } \\
\qquad(\mathrm{N}=142 \text { features) }\end{array}$} \\
\hline & \multicolumn{2}{|c|}{$\begin{array}{l}\text { Perspective of Adults with } \\
\text { Neurological Disability Only } \\
\text { ( } n=93 \text { features) }\end{array}$} & \multicolumn{2}{|c|}{$\begin{array}{l}\text { Perspective of Family / } \\
\text { Non-family Carers Only } \\
\text { ( } n=7 \text { features) }\end{array}$} & \multicolumn{2}{|c|}{$\begin{array}{c}\text { Perspective of Adults with } \\
\text { Disability \& Carers } \\
\text { (Commonly Reported Features) } \\
\text { ( } n=22 \text { features) }\end{array}$} & \multicolumn{2}{|c|}{$\begin{array}{c}\text { Observational Evidence } \\
\text { (Case Series Design) } \\
(n=20 \# \text { features })\end{array}$} \\
\hline $\begin{array}{l}\text { The me 2: } \\
\text { A Homely Environment } \\
\text { ( } n=18 \text { unique features; } n=19 \\
\text { total features) }\end{array}$ & $4^{*}$ & 4 & 5 & 71 & 9 & 41 & 0 & 0 \\
\hline $\begin{array}{l}\text { Theme 4: } \\
\text { Privacy ( } n=7 \text { features) }\end{array}$ & 4 & 4 & 1 & 14 & 2 & 9 & 0 & 0 \\
\hline
\end{tabular}




\begin{tabular}{|c|c|c|c|c|c|c|c|c|}
\hline \multirow[t]{3}{*}{ Theme } & \multicolumn{8}{|c|}{$\begin{array}{l}\text { Housing Design Features Identified } \\
\qquad(\mathrm{N}=142 \text { features })\end{array}$} \\
\hline & \multicolumn{2}{|c|}{$\begin{array}{l}\text { Perspective of Adults with } \\
\text { Neurological Disability Only } \\
\qquad(n=93 \text { features) }\end{array}$} & \multicolumn{2}{|c|}{$\begin{array}{l}\text { Perspective of Family / } \\
\text { Non-family Carers Only } \\
\qquad(n=7 \text { features) }\end{array}$} & \multicolumn{2}{|c|}{$\begin{array}{c}\text { Perspective of Adults with } \\
\text { Disability \& Carers } \\
\text { (Commonly Reported Features) } \\
\text { ( } n=22 \text { features) }\end{array}$} & \multicolumn{2}{|c|}{$\begin{array}{c}\text { Observational Evidence } \\
\text { (Case Series Design) } \\
(n=20 \# \text { features })\end{array}$} \\
\hline & $n$ & $\%$ & $n$ & $\%$ & $n$ & $\%$ & $n$ & $\%$ \\
\hline $\begin{array}{l}\text { Theme 5: } \\
\text { Supportive Care Features } \\
\text { ( } n=4 \text { features) }\end{array}$ & 2 & 2 & 0 & 0 & 2 & 9 & 0 & 0 \\
\hline $\begin{array}{l}\text { Theme 6: } \\
\text { Rehabilitation and Exercise } \\
\text { ( } n=2 \text { features) }\end{array}$ & 2 & 2 & 0 & 0 & 0 & 0 & 0 & 0 \\
\hline Total: & 93 & 100 & 7 & 100 & 22 & 100 & 20 & 100 \\
\hline
\end{tabular}

Note. +1 feature also relates to Neighbourhood Theme 1 (physical access to public places and spaces)

* 4 unique features identified; 5 features identified in total, where 1 feature was previously identified in Theme 1 and also relates to Theme 3

$\wedge 9$ unique features identified; 17 features identified in total, where 8 features were previously identified in Theme 1 or Theme 2

\# 20 unique features identified; 21 features identified in total, where 1 feature was previously identified in Housing Design Theme 1 (physical accessibility sub-theme) and 9 features also relate to Neighbourhood Theme 1 (physical access to public places and spaces) 


\section{Theme 1: Physical access}

Most ( $n=102 ; 72 \%$ ) housing design features reported by the included studies related to the first theme of physical access. This finding is not surprising given that more than half of Sample 1 comprised wheelchair users and all observational (case series) evidence related to physical access. According to participants in the included studies, features that enhance the person's capacity to move within and around their home improves independence and autonomy given that they are less reliant on the assistance of others. Housing features that promote (rather than hinder) the person's physical access are therefore important to include in housing for adults with neurological disability. The 102 features that enhanced or hindered the person's physical access at home reflected the following three sub-themes: (a) physical accessibility; (b) ease of access to household items or resources; and (c) technological features. Each sub-theme is briefly described below.

'Physical accessibility' sub-theme. Features comprising the physical accessibility sub-theme were seen to facilitate or impede the person's physical movement through space. For example, ramps with an appropriate inclination (i.e., not too steep) (study \#1, \#12, \#13, $\# 15, \# 17, \# 23, \# 26$ ) or a level entry (\#3, \#4) enable the person to enter or exit their dwelling unassisted. Similarly, a narrow doorway (\#4, \#6) or an incorrectly placed handrail (\#9) may hinder the person's movement through their home. Indeed, outdoor features that facilitate (e.g., smooth level concrete [\#23,\#26]) or impede (e.g., grass [\#23]) physical accessibility were also identified. In all, 53 (52\%) of the 102 physical access features mentioned in the included studies comprised the 'physical accessibility' sub-theme.

'Ease of access to household items or resources' sub-theme. The second physical access sub-theme comprised features that improved or obstructed the person's ease of access to household items or resources. For example, a bedside table that is the same height as the bed (\#15) enables the person to reach items placed on their bedside table (access to household 
items), and mixer taps (i.e., lever taps [\#15]) facilitates the person's ease of access to water (access to resources). As one non-family paid carer in study \#9 explains, "Everything is at a level, nothing is too high, so they can independently do it themselves” (p. 2154). Housing design features that obstruct the person's ease of access to household items or resources, such as unreachable cupboards / shelving (\#9,\#21) and inaccessible kitchen sinks (\#9) are of no use to adults with neurological disability. A total of 25 (25\%) of the 102 physical access features identified comprised this sub-theme.

'Technological features' sub-theme. The third and final physical access sub-theme comprised technological features that were seen to facilitate the person's physical accessibility (sub-theme 1) or ease of access to household items or resources (sub-theme 2) within their home. For example, automated windows (\#16), automated lights (\#16), and a stove guard in the kitchen (\#20) were viewed positively by adults with neurological disability in the included studies. However, several technological features that were considered difficult to use or caused irritation in use were also identified. These negative features were described as hindering the person's physical accessibility (sub-theme 1) or ease of access to household items or resources (sub-theme 2). Whether the technological features were considered positive or negative in nature largely depended on the person's degree of functionality (i.e., ability to use the feature/s). Three features in particular - automated doors (\#8, \#12, \#13,\#16), automatic faucets (\#8, \#20), and security alarms (\#8, \#20) - were considered useful by some people with neurological disability, but problematic by others. Twenty-four (24\%) of the 102 physical access features identified comprised this sub-theme. Interestingly, adults with neurological disability in the included studies mentioned 22 of the 24 technological features identified. 


\section{Theme 2: A homely environment}

In all, 18 (13\%) of the 142 housing design features identified reflected the second key theme, a homely environment. According to adults with neurological disability and their carers, housing that encompasses a homely, domestic feel rather than an institutional or a hospitallike atmosphere not only increases the person's sense of belonging, it also represents a place friends and family enjoy, and are therefore encouraged to visit. According to participants in the selected studies, example features that promote a homely environment include smaller, intimate dining tables (rather than long impersonal dining tables) (\#9), features that promote social connectedness with friends and family (e.g., social outdoor spaces, including a barbeque area [\#9]), bright interior design (\#2), and no institutional elements on the front door (e.g., security codes [\#9]). Interestingly, although security codes may enhance the person's physical access (i.e., ease of entry into dwelling) and sense of security (discussed in the next section), security codes may also reflect institutional elements and should be avoided, according to some adults with neurological disability in the included studies. A quieter, less busy environment, compared to what was experienced in residential aged care (\#2, \#7), was also viewed as positive by some but negative by others. These findings highlight the multifaceted interpretation of homeliness and the need to individualise residential environments for adults with neurological disability.

Theme 3: Resident safety and security

Nine (6\%) of the 142 housing design features identified reflected the third key theme, resident safety and security. Interestingly, adults with neurological disability in the selected studies identified all but one feature related to this theme. The reported features either enhanced or compromised: (a) the person's safety within the dwelling (e.g., non-slip tiles in the bathroom [\#15]); (b) their protection from others (e.g., security alarm [\#8,\#20]); or (c) their protection from the elements (e.g., weather and seasons [\#4]). Features that improved 
the person's safety and security at home not only protected the person from danger, they also boosted the person's psychological wellbeing. It is important to note that eight safety and security features were reported by those who participated in one research study (\#15). These findings should therefore be further explored and substantiated.

Theme 4: Privacy

Seven (5\%) of the 142 housing design features reported in the selected studies related to the fourth key theme, privacy. Features that promoted the person's privacy enhanced the person's psychological wellbeing, according to the participants in the included studies. Example privacy features included the person having access to their own bedroom (rather than sharing a bedroom with others) (\#2,\#11), a separate lounge room so the person did not have to entertain their visitors in their bedroom (\#2, \#11), private outdoor space to connect with nature (e.g., balcony area [\#9]), and an ensuite bathroom (\#16, \#18). Conversely, limited circulation spaces in the bathroom / toilet area for wheelchair users (\#2, \#4, \#12) made it difficult (and sometimes impossible) to close the bathroom / toilet door. With the exception of a confined bathroom / toilet area, the aforementioned features reported by Sample 1 and Sample 2 in the selected studies are notable in housing design for adults with neurological disability.

\section{Theme 5: Supportive care features}

Four (3\%) of the 142 design features identified reflected the fifth key theme, supportive care features. According to Sample 1 and Sample 2 in the selected studies, inclusion of these features improved (a) the comfort of adults with neurological disability and their carers; (b) client and carer workplace health and safety; and (c) the availability and efficiency of the care provided. For example, participants suggested air-conditioning units in the bedroom (\#16) and living room (\#16) were important to include in housing design. Air-conditioning units increased the person's (and carers') comfort and maintained the person's body temperature 
levels at the required (safe) temperature. This is an important consideration given that internal mechanisms responsible for body temperature regulation are commonly affected by neurological injury or illness (Gaither et al., 2015). In addition, the selected studies identified that both samples supported the installation of a ceiling / tracking hoist (\#16, \#19), and a spare bedroom for a non-family paid carer to stay overnight (\#19). The inclusion of a ceiling / tracking hoist improved carer workplace health and safety and care efficiency by simplifying the hoist transfer process, and a spare bedroom for non-family paid carers increases the person's access to their carers, if needed (i.e., provides a 'safety net' that may put the person at ease).

\section{Theme 6: Rehabilitation and exercise}

The final two (1\%) design features identified related to the sixth theme, rehabilitation and exercise. Adults with neurological disability in the included studies identified both features; an outdoor swimming / hydrotherapy pool (\#2, \#9, \#12) and a therapy centre (i.e., access to gym equipment [\#9]). The inclusion of these features in housing design made it easier for people with disability to engage in rehabilitation and exercise, thereby enabling the person to improve and/or maintain their physical health. As a participant in study \#2 explained,

Someone takes me to the pool. His job is, or one of his jobs is trying to keep me

fit...We are doing kicking, because I live in the chair full-time and I starting [sic]

kicking a couple of months ago...I was very surprised in kicking myself [sic] (p. 96). The inclusion of housing features that facilitated rehabilitation and exercise at home appeared to benefit adults with neurological disability, given that ongoing rehabilitation is often required once the person is discharged from hospital to home (in the case of an acquired neurological injury) (Foster et al., 2012). 


\section{Location}

The 12 location features identified related to three key themes: (1) proximity to local amenities; (2) proximity to social networks / support; and (3) proximity to local services. These themes confirm the importance of community-based resources that are situated closeby, which promotes less commute time, less expenditure on travel, and greater flexibility regarding access times. Table 4 presents an overview of the results. No location features related to more than one theme. 
Table 4

Number and Percentage of Location Features Reported by the 26 Included Studies

\begin{tabular}{|c|c|c|c|c|c|c|c|c|}
\hline \multirow[t]{2}{*}{ Theme } & \multicolumn{8}{|c|}{$\begin{array}{l}\text { Location Features Identified } \\
\qquad(\mathrm{N}=12 \text { features })\end{array}$} \\
\hline & \multicolumn{2}{|c|}{$\begin{array}{l}\text { Perspective of Adults with } \\
\text { Neurological Disability Only } \\
\text { ( } n=4 \text { features) }\end{array}$} & \multicolumn{2}{|c|}{$\begin{array}{l}\text { Perspective of Family / } \\
\text { Non-family Carers Only } \\
\text { ( } n=0 \text { features) }\end{array}$} & \multicolumn{2}{|c|}{$\begin{array}{c}\text { Perspective of Adults with } \\
\text { Disability \& Carers } \\
\text { (Commonly Reported Features) } \\
\text { ( } n=8 \text { features) }\end{array}$} & \multicolumn{2}{|c|}{$\begin{array}{c}\text { Observational Evidence } \\
\text { (Case Series Design) } \\
\text { ( } n=0 \text { features })\end{array}$} \\
\hline $\begin{array}{l}\text { Theme 2: } \\
\text { Proximity to Social Networks and } \\
\text { Support ( } n=4 \text { features) }\end{array}$ & 1 & 25 & 0 & 0 & 3 & 37.5 & 0 & 0 \\
\hline Total: & 4 & 100 & 0 & 0 & 8 & 100 & 0 & 0 \\
\hline
\end{tabular}


Theme 1: Proximity to local amenities

Five (42\%) of the 12 location features identified related to the first key theme, proximity to local amenities. According to Sample 1 and Sample 2 within one selected study (\#19), housing that was within walking (or wheeling) distance to a café, shopping centre, Church, bank, and library was most convenient. The findings from study \#19 should be interpreted with caution however, as no reporting distinction was made in this research paper to differentiate the views of Sample 1 and Sample 2. Further research is needed to identify potential differences in opinion regarding the local amenities deemed important for adults with neurological disability to live near.

Theme 2: Proximity to social networks / support Four (33\%) of the 12 location features identified by the selected studies reflected the second key theme, proximity to social networks and support. According to participants in the selected studies, the person's housing location affected their access to social networks (and therefore social support) in their community. The person's proximity to their social networks (e.g., friends [\#6, \#11, \#19]; relatives, including children [\#1, \#6, \#11, \#17,\#19]) can be seen as an important consideration for housing for adults with neurological disability.

Theme 3: Proximity to local services

Adults with neurological disability in the included studies viewed good proximity to services in the local community in a positive way. In all, three (25\%) of the 12 location features identified reflected this third and final theme. According to Sample 1, living near the following services was ideal: public transport (\#1, \#11, \#14,\#17), the person's place of employment (\#14), and medical services (\#1,\#14). Good proximity to local services must therefore be properly considered when designing suitable housing for adults with neurological disability. 


\section{Neighbourhood}

The 54 neighbourhood features reported in the included studies related to three key themes:

(1) physical access to public places and spaces; (2) provision of adequate community services and resources; and (3) provision of appropriate social networks / support. These themes endorse the interconnected nature of housing with social networks, and provide a more detailed understanding of the preferred physical, social, and/or economic nature of local communities than previously reported. Table 5 presents an overview of the results, where 10 features previously identified in Housing Design Theme 1 (physical access: physical accessibility sub-theme) also related to the neighbourhood context. 
Table 5

Number and Percentage of Neighbourhood Features Reported by the 26 Included Studies

\begin{tabular}{|c|c|c|c|c|c|c|c|c|}
\hline \multirow[t]{2}{*}{ Theme } & \multicolumn{8}{|c|}{$\begin{array}{l}\text { Neighbourhood Features Identified } \\
\qquad(\mathrm{N}=54 \text { features })\end{array}$} \\
\hline & \multicolumn{2}{|c|}{$\begin{array}{l}\text { Perspective of Adults with } \\
\text { Neurological Disability Only } \\
\qquad(n=44 \text { features) }\end{array}$} & \multicolumn{2}{|c|}{$\begin{array}{l}\text { Perspective of Family / } \\
\text { Non-family Carers Only } \\
\text { ( } n=0 \text { features) }\end{array}$} & \multicolumn{2}{|c|}{$\begin{array}{c}\text { Perspective of Adults with } \\
\text { Disability \& Carers } \\
\text { (Commonly Reported Features) } \\
\text { ( } n=1 \text { feature) }\end{array}$} & \multicolumn{2}{|c|}{$\begin{array}{c}\text { Observational Evidence } \\
\text { (Case Series Design) } \\
(n=9 \wedge \text { features })\end{array}$} \\
\hline $\begin{array}{l}\text { Theme 2: } \\
\text { Provision of Community Services } \\
\text { and Resources ( } n=13 \text { features) }\end{array}$ & 12 & 27 & 0 & 0 & 1 & 100 & 0 & 0 \\
\hline Total: & 44 & 100 & 0 & 0 & 1 & 100 & 9 & 100 \\
\hline
\end{tabular}

Note. *1feature was previously identified in Housing Design theme 1 (physical access: physical accessibility sub-theme)

$\wedge$ All nine features were previously identified in Housing Design Theme 1 (physical access: physical accessibility sub-theme) 
Theme 1: Physical access to public places and spaces

Similar to the design of the person's home, most ( $n=30 ; 56 \%)$ neighbourhood features identified related to physical access. Interestingly, family/non-family carers did not report any features associated with this theme. Neighbourhood features that promoted physical accessibility, according to adults with neurological disability in the selected studies, included a flat neighbourhood (\#1) and wheelchair friendly footpaths (\#1). Conversely, physically inaccessible buildings (\#3, \#4, \#6, \#8, \#12,\#13) and public bathrooms that do not accommodate wheelchair users (\#3,\#13) were example neighbourhood features that emphasized the person's socially constructed physical limitations. Indeed, the observational (case series) evidence that identified nine neighbourhood features relating to physical access in the community also supported these findings.

Theme 2: Provision of adequate community services and resources In all, 13 (24\%) of the 54 neighbourhood features identified by participants in the included studies reflected the second key theme, provision of adequate community services and resources. Participants described 'adequacy' as meeting the needs of adults with neurological disability. According to the participants in the selected studies, neighbourhoods either provided or neglected to provide suitable services (e.g., physically accessible, affordable, and reliable public transport [\#1, \#11,\#14,\#17]; an important consideration given that neurological impairment often comprises the ability of adults to drive [Bivona et al., 2012; Ponsford et al., 2014]) and resources (e.g., disability-specific equipment [\#1]). This, in turn, impacted their experiences of community living and ability to independently engage with their community. Housing for adults with neurological disability should therefore be constructed in neighbourhoods that facilitate the person's access to relevant services and resources. 
Theme 3: Provision of appropriate social networks / support

The final 11 (20\%) neighbourhood features identified by the included studies related to the third and final theme, provision of appropriate social networks and support. According to adults with neurological disability, certain neighbourhood features influenced the person's access to local support systems. For example, living in a neighbourhood with friendly and helpful neighbours (\#1, \#5, \#11, \#14) or in an area where able-bodied community members interact with individuals with a disability in a normalised way (\#7) increased the person's access to supportive social networks. In contrast, living in a perceived unsafe neighbourhood $(\# 1, \# 6)$ or in an area less accepting of people with a disability (\#3, \#4, \#5, \#6, \#7, \#10) thwarted the person's access to supportive social networks. Participants in the selected studies therefore highlighted the fact that simply living in the community did not necessarily guarantee participation or a sense of belonging. Thus, access to appropriate social networks and support remains an important component in the development of suitable housing for adults with neurological disability.

\section{Discussion}

This review aimed to identify and synthesise studies published post-2003 that could inform housing design and development decisions for adults between 18 years and 65 years of age with neurological disability. Specifically, the review sought to identify housing design, location, and neighbourhood features that could inform a broader understanding of housing preferences for adults with neurological disability.

The evidence from this review was sourced from 26 eligible studies. The majority of the studies employed a qualitative research design, indicating that interviews, focus groups, and case study research approaches are used most often to investigate housing features specific to the target population. Across the studies, the research predominantly explored the views of adults with neurological disability, and to a much lesser extent, their family and 
non-family carers (see Table 6). In relation to the specific housing needs of adults with neurological disability, the findings of this review confirmed that physical access (at home and in the community), together with health and safety, are indeed important attributes to inform housing development. However, the findings have also highlighted additional aspects of housing (e.g., homely atmosphere; privacy; comfort; efficiency of care; convenience of local amenities, nearby social networks, good proximity to local services, adequacy of community services and resources, and appropriate community support systems) that ought to inform housing solutions for adults with neurological disability. Indeed, housing elements that should not inform housing solutions (e.g., technology that is difficult to use, living in an isolated location or in an area that lacks community resources, poor community acceptance, and perceived unsafe neighbourhoods) were also identified. These findings extend dominant understandings of suitable housing beyond physical access and health and safety concerns. Future housing provision that fails to incorporate this broader understanding of housing suitability into early design processes risk affecting the long-term biopsychosocial health and wellbeing of adults with neurological disability and the family/non-family carers who also interact with the residential environment.

Table 6 also highlights that the majority of the housing features identified related to design (incorporating safety and accessibility) and the neighbourhood setting. Less research explored specific housing location features. Similarly, there was limited observational (case series design) evidence available; specifically, limited in number (e.g., only five eligible studies) and in diversity (e.g., all five studies focused on physical accessibility). Although this systematic review has identified important features that can contribute to a set of housing preferences for adults with neurological disability, these aforementioned issues limit the completeness of the reported evidence. 
Table 6

Number of Housing Features Reported by Respective Participant Groups and Observational (Case Series Design) Evidence

\begin{tabular}{|c|c|c|c|c|}
\hline \multirow[t]{2}{*}{ Source } & \multicolumn{4}{|c|}{ Housing Features Identified } \\
\hline & $\begin{array}{l}\text { Housing Design } \\
\text { ( } n=142 \text { features) }\end{array}$ & $\begin{array}{c}\text { Location } \\
(n=12 \text { features })\end{array}$ & $\begin{array}{l}\text { Neighbourhood } \\
\text { ( } n=54 \text { features) }\end{array}$ & $\begin{array}{l}\text { Total Features by Source } \\
\text { (N=198 unique features) }\end{array}$ \\
\hline Family/non-family carers (Sample 2 only) & 7 & 0 & 0 & 7 \\
\hline $\begin{array}{l}\text { Adults with neurological disability \& family/non-family carers } \\
\text { (Sample } 1 \text { \& Sample 2) (i.e., commonly reported features) }\end{array}$ & 22 & 8 & 1 & 31 \\
\hline
\end{tabular}

Note. * One neighbourhood feature identified by adults with neurological disability (Sample 1 only) also related to housing design, and should therefore not be included in the count of unique housing features identified.

$\wedge$ The nine neighbourhood features identified by observational (case series design) evidence also related to housing design, and should therefore not be included in the count of unique housing features identified. 


\section{Limitations and future research directions}

Although this review was conducted systematically and comprehensively, several limitations of this review must be noted. First, the inconsistent (or absence of) demographic reporting in a small number of included studies limits the generalizability of the review findings. On a related note, studies focusing on the housing needs of individuals diagnosed with Dementia or Alzheimer's disease were excluded from this review, since these conditions are particularly prevalent amongst the elderly (and therefore beyond the scope of this review) (Alzheimer's Association, 2015; WHO, 2012). However, given that a relatively small proportion (yet still significant number) of people aged under 65 years have been diagnosed with Dementia (i.e., between 2\% and 10\% of all global diagnoses [WHO, 2012]) or Alzheimer's disease (i.e., approximately 200,000 Americans ${ }^{3}$ [Alzheimer's Association, 2015]), future reviews could reasonably investigate the housing needs of this group. Future research could also focus on housing in non-English speaking cultures, to provide a more detailed understanding of culturally appropriate residential environments.

Second, according to the general housing classification framework applied, much of the research focused on housing features related to housing design and the neighbourhood setting with limited or no focus on housing location. This is despite the equal inclusion of keywords relating to all three factors in the search matrix (scholarly literature), and the equal exclusion of keywords relating to all three factors when searching the grey literature (i.e., only the term 'disability' was entered into the search bar for two websites, and all published reports post-2003 were downloaded from the third website). This finding highlights that research in this field needs to explore housing location features in greater detail, given that the person's proximity to available community resources directly affects their wellbeing by enhancing or hindering their physical access to needed resources (Schulz \& Northridge,

\footnotetext{
${ }^{3}$ Only American prevalence rates specific to Alzheimer's disease were available.
} 
2004; Shaw, 2004). Further investigation of the perspective of family/non-family carers is also important, given that the voice of this group was also largely missing from the available evidence.

It was also notable that the studies reviewed inferred that with whom the person lives and how they are supported at home in terms of their healthcare needs were additional considerations to housing design, location, and neighbourhood. Research that deliberately explores consumer experiences and preferences relating to aspects of residential tenancy and healthcare support will no doubt contribute a more accurate picture of what people with neurological disability want from their housing and why. Research of this nature will also facilitate the expansion of frameworks of understanding when considering housing needs for people with neurological disability.

\section{Implications for practice}

The findings of this review provide a detailed understanding of housing features that might inform future principles for housing design and development for younger adults with neurological disability. Importantly, the review findings provide example housing features so that stakeholders involved in housing design and development (e.g., architects, designers, builders, developers, funding agencies) have access to practical information to inform housing decisions. Preliminary guiding principles relating to housing design, location, and the neighbourhood context for inclusive housing solutions have been developed from this review, and include the following:

- Housing Design:

o Housing for adults with neurological disability ought to: (a) facilitate physical access (e.g., physical accessibility; ease of access to household items and resources; technological features) inside and outside the person's home, (b) encompass a homely environment, (c) enhance the person's safety, security, 
and privacy (while not compromising the homely environment), (d) improve the comfort of users, client and carer workplace health and safety, and the availability and efficiency of the care provided, and (e) provide access to rehabilitation and exercise facilities.

- Location:

o Housing for adults with neurological disability ought to be located within good proximity to local amenities, the person's social networks, and local services.

- Neighbourhood:

o Housing for adults with neurological disability ought to be constructed in neighbourhoods that: (a) promote physical access to public places and spaces, (b) provide suitable community services and resources for adults with neurological disability, and (c) enable the development of positive social networks, thereby facilitating social support.

Although a noteworthy contribution to the literature, limitations of these preliminary guiding principles for policy and practice, including the ambiguities of concepts such as 'homely environment' and the complexities involved in enabling 'positive social networks', must be noted. Future research conducted to address the limitations of this review will further develop these guiding principles and associated example housing features.

\section{Conclusion}

This systematic review summarized the findings of 26 eligible studies that reported 198 unique housing features that could inform inclusive housing design and development. The review clearly identified safety and accessibility as essential design elements, but importantly identified a number of housing features reflecting the broader context of where and how people live. For example, the findings indicated that housing features that promote a homely 
atmosphere, privacy, comfort, efficiency of care, convenience of local amenities, nearby social networks, good proximity to local services, adequacy of community services and resources, and appropriate community support systems ought to inform future housing solutions for younger adults with neurological disability. In contrast, technology that is difficult to use, living in an isolated location or in an area that lacks community resources, poor community acceptance, and perceived unsafe neighbourhoods are example features that should not inform future housing development opportunities for this population. Consumers with disability and all other stakeholders involved in the design and development of public and private inclusive housing can benefit from the practical findings reported in this study. 


\section{References}

Accessibility for Ontarians Act 2005, S. O. 2005, Chapter 11. Retrieved from https://www.ontario.ca/laws/statute/05a11

Alzheimer's Association, 2015. 2015 Alzheimer's disease facts and figures. Alzheimers Dement 11 (3), 332-384. doi:10.1016/j.jalz.2015.02.003

Australian Department of Human Services, n.d. About the NDIS. Retrieved from https://www.ndis.gov.au/html/sites/default/files/documents/About-us/AbouttheNDIS.pdf

Australian Network for Universal Housing Design, RI Australia, 2014. Report on the Progress of the National Dialogue on Universal Housing Design 2010-2014. Authors, place of publication unknown.

Ballin, L., Balandin, S., 2007. An exploration of loneliness: communication and the social networks of older people with cerebral palsy. Journal of Intellectual \& Developmental Disability 32 (4), 315-327. doi:10.1080/13668250701689256.

Bell, S.S., 2012. Librarian’s Guide to Online Searching (3rd ed.). ABC-CLIO, Santa Barbara, CA.

Bivona, U., D’Ippolito, M., Giustini, M., Vignally, P., Longo, E., Taggi, F., Formisano, R., 2012. Return to driving after severe traumatic brain injury: Increased risk of traffic accidents and personal responsibility. Journal of Head Trauma Rehabilitation 27 (3), 210-215. doi:10.1097/HTR.0b013e31822178a9

Boman, I., Tham, K., Granqvist, A., et al., 2007. Using electronic aids to daily living after acquired brain injury: a study of the learning process and the usability. Disability \& Rehabilitation: Assistive Technology 2 (1), 23-33.

doi:10.1080/174831006008562013 
Bozzolini, G., Cassibba, S., 2008. Improving home accessibility for a person with a disability after spinal cord injury. European Journal of Physical and Rehabilitation Medicine 44 (4), 455-459.

Canada Mortgage and Housing Corporation, 2012. Canadian Housing Observer 2012 (10th ed.). Retrieved from https://www.cmhc-schl.gc.ca/odpub/pdf/67708.pdf

Cardol, M., de Haan, R.J., van den Bos, G.A., et al., 1999. The development of a handicap assessment questionnaire: The impact on participation and autonomy (IPA). Clinical Rehabilitation 13 (5), 411-419. doi:10.1191/026921599668601325

Carlson, C., Aytur, S., Gardner, K., et al., 2012. Complexity in built environment, health, and destination walking: A neighborhood-scale analysis. Journal of Urban Health: Bulletin of the New York Academy of Medicine 89 (2), 270-284. doi:10.1007/s11524-011-9652-8

Commonwealth of Australia, 2016. Ageing and Aged Care: Commonwealth HACC Program. Retrieved from https:/agedcare.health.gov.au/programsservices/commonwealth-home-support-programme/commonwealth-hacc-program

Commonwealth of Australia, 2015. Community Affairs References Committee: Adequacy of Existing Residential Care Arrangements Available for Young People with Severe Physical, Mental, or Intellectual Disabilities in Australia (Senate Report). Retrieved from http^/www.aph.gov.au/Parliamentary_Business/Committees/Senate/Community_Affa irs/Young_people_in_aged_care/Report

Commonwealth of Australia, 2003. Third Commonwealth State/Territory Disability Agreement. Retrieved from https://www.dss.gov.au/sites/default/files/documents/05_2012/cstda3may07.pdf 
Cummins, S., Curtis, S., Diez-Rouz, A.V., et al., 2007. Understanding and representing 'place' in health research: A relational approach. Soc Sci Med 65 (9), 1825-1838. doi:10.1016/j.socscimed.2007.05.036

Dattilo, J., Estrella, G., Estrella, L.J., et al., 2008. "I have chosen to live life abundantly": perceptions of leisure by adults who use augmentative and alternative communication. AAC: Augmentative \& Alternative Communication 24 (1), 16-28. doi:10.1080/07434610701390558.

Dickson, A., Ward, R., O'Brien, G., et al, 2011. Difficulties adjusting to post-discharge life following a spinal cord injury: an interpretative phenomenological analysis. Psychology, Health \& Medicine 16 (4), 463-474. doi:10.1080/13548506.2011.555769

Dutta, T., King, E.C., Holliday, P.J., et al., 2011. Design of built environments to accommodate mobility scooter users: part I. Disability \& Rehabilitation: Assistive Technology 6 (1), 67-76. doi:10.3109/17483107.2010.509885

Foster, A.M., Armstrong, J., Buckley, A., Sherry, J., Young, T., Foliaki, S., James-Hohaia, T.M., Theadom, A., McPherson, K.M., 2012. Encouraging family engagement in the rehabilitation process: A rehabilitation provider's development of support strategies for family members of people with traumatic brain injury. Disability and Rehabilitation 34(22), 1855-1862. doi:10.3109/09638288.2012.670028

Gaither, J.B., Galson, S., Curry, M., et al., 2015. Environmental hyperthermia in prehospital patients with major traumatic brain injury. The Journal of Emergency Medicine 49 (3), 375-381. doi:10.1016/j.jemermed.2015.01.038

Grant, E., Chong, A., Zillante, G., et al., 2014. The NDIS, housing and Indigenous Australians living with a disability. Parity 27 (5), 25-26. 
Hempel, D.J., Tucker Jr, L.R., 1979. Citizen preferences for housing as community social indicators. Environment and Behavior 11 (3), 399-428. doi:10.1177/0013916579113005

Higgins, J.P.T., Green, S. (Eds), 2011. Cochrane Handbook for Systematic Reviews of Interventions (Version 5.1.0). The Cochrane Collaboration. Retrieved from http://www.cochrane-handbook.org

Hoppestad, B.S., 2006. Essential elements for assessment of persons with severe neurological impairments for computer access utilizing assistive technology devices: A Delphi study. Disability and Rehabilitation 1 (1-2), 3-16. doi:10.1080/09638280500167019

Hurd, W.J., Morrow, M.M., Kaufman, K.R., et al., 2008. Influence of varying level terrain on wheelchair propulsion biomechanics. Am J Phys Med Rehab 87 (12), 984-991. doi:10.1097/PHM.0b013e31818a52cc

Hurd, W.J, Morrow, M.M., Kaufman, K.R., et al., 2009. Wheelchair propulsion demands during outdoor community ambulation. Journal of Electromyography and Kinesiology 19 (5), 942-947. doi:10.1016/j.jelekin.2008.05.001

Iwarsson, S., Slaug, B., 2010. The Housing Enabler: A Method for Rating/Screening and Analysing Accessibility Problems in Housing: Manual for the Complete Instrument and Screening Tool. Veten \& Skapen HB and Slaug Data Management AB, Lund and Staffanstorp, Sweden.

Kauko, T., 2006. What makes a location attractive for the housing consumer? Preliminary findings from metropolitan Helsinki and Randstad Holland using the analytical hierarchy process. Journal of Housing and the Built Environment 21(2), 159-176. doi:10.1007/s10901-006-9040-y 
Koontz, A.M., Brindle, E.D., Kankipati, P., et al., 2010. Design features that affect the maneuverability of wheelchairs and scooters. Arch Phys Med Rehab 91(5), 759-764. doi:10.1016/j.apmr.2010.01.009

Koontz, A.M., Cooper, R.A., Boninger, M.L., et al., 2005. A kinetic analysis of manual wheelchair propulsion during start-up on select indoor and outdoor surfaces. Journal of Rehabilitation Research and Development 42(4), 447-458. doi:10.1682/JRRD.2004.08.0106

Krieger, J., Higgins, D.L., 2002. Housing and health: Time again for public health action. American Journal of Public Health 92(5), 758-768. doi:10.2105/AJPH.92.5.758

Kroehn, M., Hutson, K., Faulkner, D., et al., 2007. The Housing Careers of Persons with a Disability and Family Members with Care Responsibilities for Persons with a Disability. Project C: Qualitative Data Collection Report of Focus Groups. Australian Housing and Urban Research Institute, Melbourne, Victoria.

Landis, J., Koch, G., 1977. The measurement of observer agreement for categorical data. Biometrics 33 (1), 159-174. doi:10.2307/2529310

Lindberg, E., Gärling, T., Montgomery, H., 1988. People’s beliefs and values as determinants of housing preferences and simulated choices. Scandinavian Housing and Planning Research 5(3), 181-197. doi:10.1080/02815738808730162

Lindén, A., Lexell, J., Lund, M.L., 2010. Perceived difficulties using everyday technology after acquired brain injury: Influence on activity and participation. Scandinavian Journal of Occupational Therapy 17 (4), 267-275. doi:10.3109/11038120903265022

Linskell, J., Bouamrane, M.M., 2012. Assisted-living spaces for end-users with complex needs: a proposed implementation and delivery model. Health Informatics Journal 18 (3), 159-170. doi:10.1177/1460458212441474 
Livable Housing Australia, 2012. Livable Housing Design Guidelines (2nd ed.). Livable Housing Australia, Sydney, Australia.

MacDonald, S., 2016. Assessment of higher level cognitive-communication functions in adolescents with $\mathrm{ABI}$ : Standardization of the student version of the functional assessment of verbal reasoning and executive strategies (S-FAVRES). Brain Injury 30 (3), 295-310. doi:10.3109/02699052.2015.1091947

Magenuka, N.S., 2006. The personal and embodied experiences of people living with a spinal cord injury in the OR Tambo district municipality in the Eastern Cape (Unpublished doctoral dissertation). University of South Africa, Pretoria, South Africa.

Mahood, Q., Van Eerd, D., Irvin, E., 2014. Searching for grey literature for systematic reviews: challenges and benefits. Research Synthesis Methods 5 (3), 221-234. doi:10.1002/jrsm.1106

Megbolugbe, I.F., Marks, A.P., Schwartz, M.B. 1991. The economic theory of housing demand: A critical review. The Journal of Real Estate Research 6(3), 381-393.

Moher, D., Liberati, A., Tetzlaff, J., et al., 2009. Preferred reporting items for systematic reviews and meta-analyses: the PRISMA Statement. BMJ 339 (7716), 332-336. doi:10.1136/bmj.b2535

Muenchberger, H., Ehrlich, C., Kendall, E., et al., 2012. Experience of place for young adults under 65 years with complex disabilities moving into purpose-built residential care. Soc. Sci. Med. 75 (12), 2151-2159. http:/dx.doi.org/10.1016/j.socscimed.2012.08.002

Nygård, L., Starkhammar, S., 2003. Telephone use among noninstitutionalized persons with dementia living alone: mapping out difficulties and response strategies. Scandinavian Journal of Caring Sciences 17 (3), 239-49. doi:10.1046/j.1471-6712.2003.00177.x 
Orrell, A., McKee, K., Torrington, J., et al., 2013. The relationship between building design and residents' quality of life in extra care housing schemes. Health \& Place 21, 5264. doi:10.1016/j.healthplace.2012.12.004

Pace, R., Pluye, P., Bartlett, G., et al., 2012. Testing the reliability and efficiency of the pilot mixed methods appraisal yool (MMAT) for systematic mixed studies review. International Journal of Nursing Studies 49 (1), 47-53. doi:10.1016/j.ijnurstu.2011.07.002

Parker, C., Barnes, S., McKee, K., et al., 2004. Quality of life and building design in residential and nursing homes for older people. Ageing and Society 24 (6), 941-962. doi:10.1017/S0144686.X04002387

Pettersson, C., Brandt, Å., Lexell, E.M., et al., 2015. Autonomy and housing accessibility among powered mobility device users. Am J Occup Ther 69 (5), 1-9. doi:10.5014/ajot.2015.015347

Pettersson, C., Iwarsson, S., Brandt, Å, et al., 2014. Men’s and women’s perspectives on using a powered mobility device: benefits and societal challenges. Scandinavian Journal of Occupational Therapy 21 (6), 438-446. doi:10.3109/11038128.2014.905634

Pettersson, C., Löfqvist, C., Malmgren, A.F., 2012. Clients’ experiences of housing adaptations: a longitudinal mixed-methods study. Disability and Rehabilitation 34 (20), 1706-1715. doi:10.3109/09638288.2012.660596

Pluye, P., Robert, E., Cargo, M., et al., 2011. Proposal: A Mixed Methods Appraisal Tool for Systematic Mixed Studies Reviews. Retrieved from http://mixedmethodsappraisaltoolpublic.pbworks.com

Ponsford, J.L., Downing, M.G., Olver, J., Ponsford, M., Acher, R., Carty, M., Spitz, G., 2014. Longitudinal follow-up of patients with traumatic brain injury: Outcome at 
two, five, and ten years post-injury. Journal of Neurotrauma 31 (1), 64-77. doi:10.1089/neu.2013.2997

Reid, D., Angus, J., McKeever, P., et al., 2003. Home is where their wheels are: experiences of women wheelchair users. Am J Occup Ther 57 (2), 186-195.

Ripat, J. D. (2011). Self-perceived participation amongst adults with spinal cord injuries: The role of assistive technology (Unpublished doctoral dissertation). The University of Manitoba, Winnipeg, Canada.

Rosenberg, L., Nygård, L., Kottorp, A., 2009. Everyday technology use questionnaire: psychometric evaluation of a new assessment of competence in technology use. OTJR: Occupation, Participation and Health 29 (2), 52-62. doi:10.3928/1539449220090301-05

Sakellariou, D., 2015. Home modifications and ways of living well. Medical Anthropology 34 (5), 456-469. doi:10.1080/01459740.2015.1012614

Saugeres, L., 2011. (Un)accommodating disabilities: housing, marginalization and dependency in Australia. Journal of Housing and the Built Environment 26 (1), 1-15. doi:10.1007/s10901-010-9201-x

Schulz, A., Northridge, M.E., 2004. Social determinants of health: implications for environmental health promotion. Health Education \& Behavior 31 (4), 455-471. doi:10.1177/1090198104265598

Scotts, M., Saville-Smith, K., James, B., 2007. International Trends in Accessible Housing for People with Disabilities: A Selected Review of Policies and Programmes in Europe, North America, United Kingdom, Japan, and Australia (Working Paper 2). Retrieved from http•//repository.digitalnz.org/system/uploads/record/attachment/361/housing_and_dis 
ability_future_proofing_new_zealand_s_housing_stock_for_an_inclusive_society__working_paper_2_.pdf

Shaw, M., 2004. Housing and public health. Annual Review of Public Health 25(1), 397-418. doi:10.1146/ annurev.publhealth.25.101802.123036

Tually, S., Beer, A., McLoughlin, P., 2011. Housing Assistance, Social Inclusion and People Living with a Disability (AHURI Final Report No.178). Australian Housing and Urban Research Institute, Melbourne, Victoria.

U.S. Department of Housing and Urban Development, 2016. Disability Rights in Housing. Retrieved from https://portal.hud.gov/hudportal/HUD?src=/program_offices/fair_housing_equal_opp/ disabilities/inhousing

Wiesel, I., Habibis, D., 2015. NDIS, Housing Assistance and Choice and Control for People with Disability (AHURI Final Report No. 258). Australian Housing and Urban Research Institute, Melbourne, Victoria.

Winkler, D., Farnworth, L., Sloan, S., et al., 2011. Moving from aged care facilities to community-based accommodation: outcomes and environmental factors. Brain Injury 25 (2), 153-168. doi:10.3109/02699052.2010.541403

Winkler, D., Holgate, N., Sloan, S., et al., 2012. The Victorian Younger People in Residential Aged Care Initiative: Evaluation of Quality of Life Outcomes for Participants. Summer Foundation Ltd, Melbourne, Victoria.

Winkler, D., Sloan, S., Callaway, L., 2007. Younger people in residential aged care: support needs, preferences and future directions. Summer Foundation Ltd, Melbourne, Victoria. 
Winkler, D., Sloan, S., Callaway, L., 2008. Younger people in residential aged care in Tasmania: characteristics, support needs and preferences. Summer Foundation Ltd, Melbourne, Victoria.

World Health Organization (WHO), 2012. Dementia: A Public Health Priority. WHO, Geneva, Switzerland.

Wright, C.J., Muenchberger, H., Whitty, J.A., 2015. The choice agenda in the Australian supported housing context: a timely reflection. Disabil. Soc. 30 (6), 834-848. http://dx.doi.org/10.1080/09687599.2015.1038336.

Yeung, P.H., Passmore, A.E., Packer, T.L., 2008. Active citizens or passive recipients: how Australian young adults with cerebral palsy define citizenship. Journal of Intellectual \& Developmental Disability 33 (1), 65-75. doi:10.1080/13668250701875129 